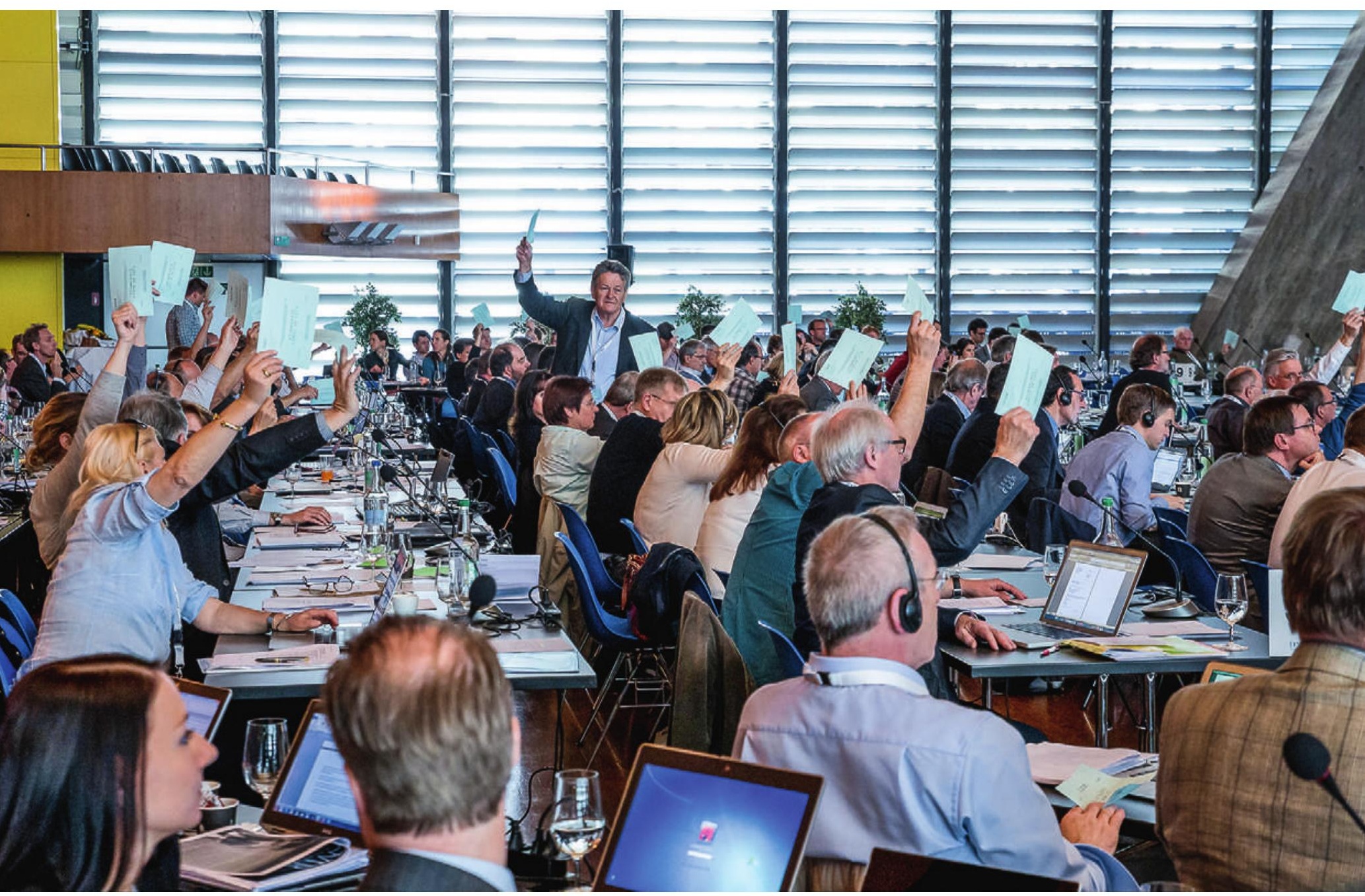

\title{
Procès-verbal de la première Chambre médicale de 2015
}

\section{Monika Henzen}

Cheffe du Secrétariat central

\section{Accueil, communications, désignation du bureau}

Jürg Schlup/président de la FMH souhaite la bienvenue aux délégués à la Chambre médicale. Il souhaite également la bienvenue aux invités, Joachim Eder/conseiller aux Etats ZG, Hans-RudolfKoelz/vice-président de l'ISFM, Jean-Pierre Keller/vice-président de l'ISFM, Beat Bär/ directeur de FMH Services, Bruno Kesseli/rédacteur en chef du BMS, Ruedi Bienz/directeur des EMH et Nicole Beutler/Furrer Hugi Partner.
D'autres invités sont également attendus: Roland Burger/société de révision BDO pour le point 3, Michel Meier/conseiller juridique de la Société de médecine du canton de Soleure pour le point 5.3.1, le Prof. Christian Kind/président de la Commission centrale d'éthique de l'ASSM pour les points 5.3.2 et 5.3.3 ainsi que le Dr Walter Gratzer et Marc Widmer/KPMG pour le point 8 .

Anne-Geneviève Bütikofer/secrétaire générale de la FMH donne les informations d'usage pour le déroulement de la séance et présente les différents documents qui ont été distribués aux délégués à leur arrivée. Elle indique 


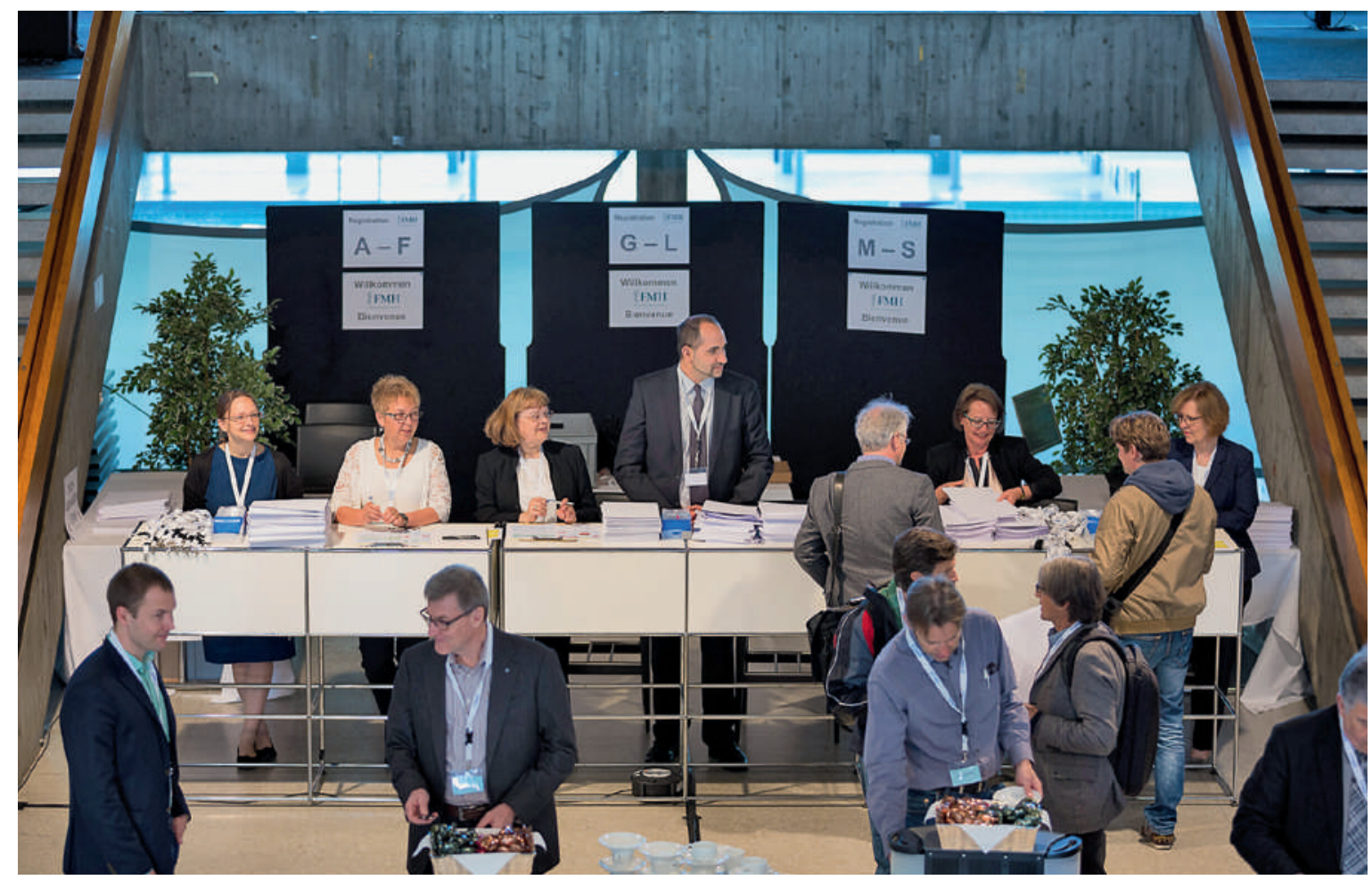

Les délégués des sociétés cantonales de médecine, des sociétés de discipline médicale et des organisations faîtières reçoivent leur bulletin de vote et les documents de séance de la part des collaborateurs de la FMH.

également que les délégués ont trouvé un questionnaire en arrivant à leur place, celui-ci leur offre la possibilité de donner leur avis sur le système de classement des documents utilisés pour télécharger les documents de séance.

Le président désigne ensuite le bureau de la Chambre médicale, qui se compose du président, du vice-président, de la secrétaire générale et des personnes suivantes qu'il propose d'élire comme scrutateurs:

Christian Bernath, Hans-Anton Vogel, Philippe Rheiner, Marcel Stampfli, Hans Ulrich Iselin, Daniel Ackermann, Hans-Jakob Riedtmann, Robert Weber, Hervé Spechbach, Daniel Schröpfer.

Les scrutateurs sont élus sans opposition.

\section{Ordre du jour}

L'ordre du jour est accepté à l'unanimité.

Jürg Schlup/président FMH demande à clore la séance au plus tard à $17 \mathrm{~h} 45$. Une proposition ayant été déposée pour clore la séance à $17 \mathrm{~h}$, les délégués procèdent au vote.

\section{Décision:}

La proposition de clore la séance à $17 \mathrm{~h} 45$ est acceptée par 63 oui contre 28 non.

Jürg Schlup/président FMH propose de ne plus prendre de décisions et de ne plus procéder à des votes après 17 h45 conformément à l'art. 11 al. 3 du Règlement d'exécution.

\section{Exposé d'introduction du président de la FMH}

Jürg Schlup/président FMH ouvre la séance de ce jour, qui se déroule sans Ernst Gähler, décédé subitement le 12 mars dernier. Vice-président de longue date, Ernst Gähler était aussi médecin, mari, père et grand-père, ainsi que député au Grand conseil du canton d'Appenzell. Le président rend hommage à cette personnalité

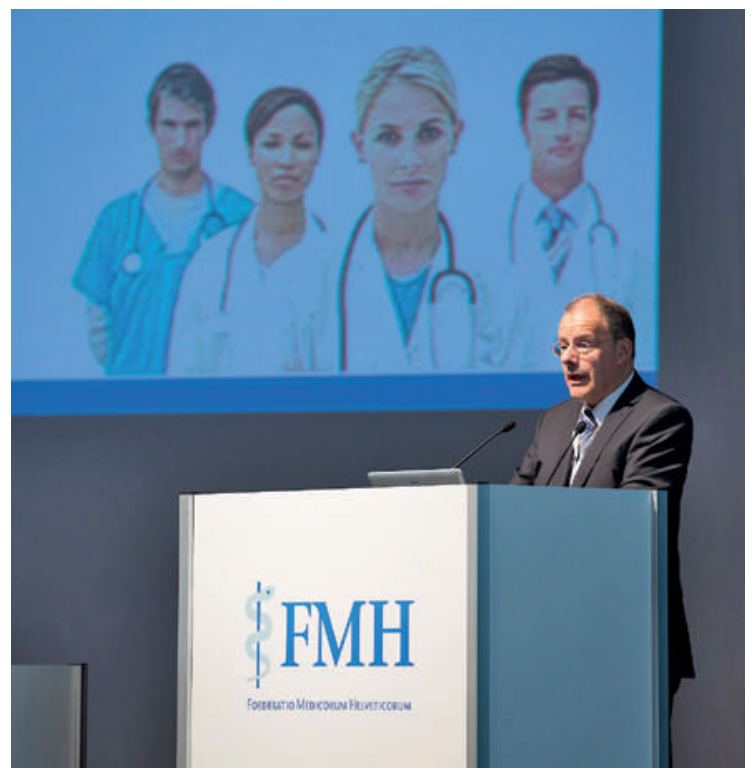

Le Dr Jürg Schlup, président de la FMH, souhaite la bienvenue aux 200 délégués à la Chambre médicale - le «parlement» de la $\mathrm{FMH}$. 
hors du commun, qui n'a jamais perdu de vue son objectif même dans les situations difficiles. Avec Ernst Gähler, la FMH perd non seulement une grande personnalité mais avant tout un ami et un homme exceptionnel. Nul doute qu'il laissera derrière lui un grand vide.

Hans-Anton Vogel/AR rend à son tour hommage à son confrère disparu avec quelques magnifiques images du canton d'Appenzell. Il se souvient de lui en tant qu'homme et ami, doué d'une grande générosité et d'un optimisme hors du commun. Ernst a toujours su trouver le bon ton pour gagner la confiance des médecins appenzellois, ce qui n'est pas chose facile. S'ils paraissent «bourrus» au premier abord, les Appenzellois n'en sont pas moins droits et intègres, et Ernst Gähler poursuivait ses objectifs de manière droite et intègre. Ernst aimait profondément son canton; son amour pour la nature, les paysages et la culture lui tenait particulièrement à cœur, car c'est de là qu'il tirait toute la force dont il avait besoin pour poursuivre ses objectifs. Infatigable comme le cours d'eau, il consacrait toute son énergie à la mission qu'il s'était fixée, toujours fidèle à sa maxime «Le secret du succès, c'est la persévérance». Ernst Gähler a marqué d'une empreinte durable la médecine de notre pays, et son décès vient nous rappeler que nous aussi, comme le disait Shakespeare, nous devons à Dieu une mort.

Jürg Schlup/président FMH rend un ultime hommage à Ernst Gähler et le remercie au nom du corps médical pour son engagement sans faille pour le bien des patients. A lui seul, Ernst possédait les quatre qualités requises pour entretenir une relation durable avec les patients: l'attention, la bienveillance, l'honnêteté et la modestie. S'il était là aujourd'hui, il n'hésiterait pas à nous encourager à poursuivre dans cette voie et à chercher ensemble des solutions viables conformément à sa fameuse maxime "Ensemble, ça va mieux et le secret du succès, c'est la persévérance».

Les délégués observent une minute de silence en mémoire du vice-président de la FMH.

Pour poursuivre le travail d'Ernst Gähler, le CC s'est réparti les différents dossiers dont il avait la charge. Urs Stoffel/CC FMH a repris la partie la plus importante du dossier des tarifs ambulatoires et se charge ainsi de la révision tarifaire. Remo Osterwalder/CC FMH a également repris une partie du même dossier «Tarifs ambulatoires», et prêtera main forte à Urs Stoffel. Le domaine «Professions paramédicales» a été repris par Gert Printzen/CC FMH et le dossier des conventions pour la médecine ambulatoire par le président.

Le président remercie les membres du CC et toute l'équipe des tarifs ambulatoires, dirigée par Kerstin Schutz, pour son engagement hors du commun dans cette période difficile.

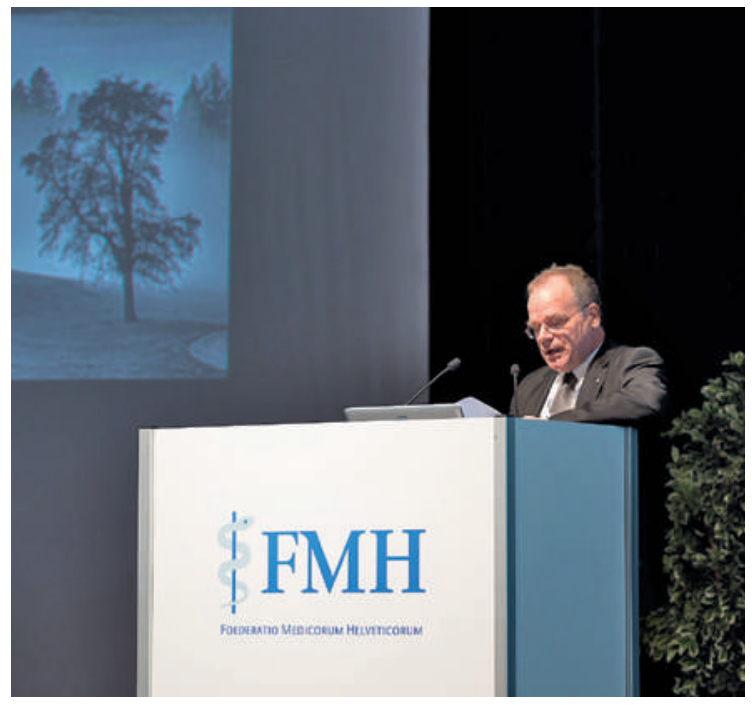

Le Dr Hans Anton Vogel, président de la société de médecine du canton d'Appenzell, rend hommage à son collègue de longue date, le Dr Ernst Gähler, vice-président de la FMH, récemment décédé.

Il en profite pour faire un bref rappel des activités politiques de la FMH au cours des derniers mois. Pour lui, la révision tarifaire reste l'un des principaux dossiers et l'un des principaux défis à relever. En 2013, la conseillère nationale Ruth Humbel a demandé au Conseil fédéral dans le cadre de l'interpellation "Adaptation de TARMED et renforcement de la médecine de famille» (13.3223) comment et selon quel calendrier il comptait faire usage de la compétence qui est la sienne en matière d'adoption du TARMED. La réponse du Conseil fédéral a été la suivante: «De ce fait, le Conseil fédéral commencera par examiner s'il convient de faire usage de sa nouvelle compétence en procédant à des adaptations ponctuelles relatives aux médecins de famille exerçant en cabinet privé et, le cas échéant les mettre en œuvre dès 2014. En revanche, l'adaptation de domaines entiers de prestations dans le TARMED demande beaucoup plus de ressources et n'est donc envisageable que dans une deuxième étape, probablement à partir de 2015.» Fait intéressant, le Conseil fédéral parle donc de deux interventions tarifaires. Par conséquent, deux scénarios sont désormais possibles pour le déroulement de la révision du tarif TARMED. Le modèle de la FMH prévoit le développement et le suivi de la structure par les partenaires tarifaires. Il est évident pour le CC quel modèle doit être poursuivi. De plus amples informations à ce sujet seront données aux délégués au point 9.

La FMH est publiquement reconnue comme un partenaire important dans le domaine de la santé. Elle entend ainsi renforcer son pouvoir d'influence. Dans le cadre du processus stratégique en cours, la FMH a réa- 
lisé l'année dernière une enquête auprès des médecins, d'une part, et des politiciens et des journalistes, d'autre part, afin de sonder son image. Les personnes interrogées avaient le choix entre une douzaine d'adjectifs différents et devaient choisir ceux qui à leurs yeux caractérisent le mieux la FMH. Un consensus s'est clairement dessiné auprès des deux groupes interrogés pour trois des quatre caractéristiques principales, tous deux décrivant la FMH comme étant sérieuse, crédible et professionnelle. La seule différence entre les deux groupes a concerné l'influence politique de la FMH: alors que le corps médical estime que la FMH dispose d'une influence relativement faible, les politiciens et les journalistes jugent au contraire que la FMH est très influente. La FMH entend rester un partenaire important tant pour relever les défis du domaine de la santé que pour solliciter le débat public sur des sujets politiques. Ses prises de position ne sont pas seulement lues, elles sont également débattues au Parlement, comme le montrent clairement les procès-verbaux des deux dernières sessions parlementaires et les invitations aux consultations parlementaires, toujours plus fréquentes. La FMH est donc présente et influente. Si cette évaluation externe est réjouissante, les participants au sondage ont également relevé nos divergences d'opinions internes et le manque de consensus. Jürg Schlup/président FMH souhaite une FMH qui évolue et s'ouvre aux changements. Il préfère évoquer l'avenir plutôt que le passé et s'investir dans des solutions objectives et susceptibles d'être mises en œuvre.

\section{Rapports annuels 2014}

\section{Communications du président de l'ISFM}

Pour Werner Bauer/président de l'ISFM, il est important que la formation postgraduée et continue bénéficie du soutien nécessaire de la Chambre médicale.

Si la formation postgraduée et continue doit rester en main des médecins, la FMH doit aussi en assumer la responsabilité. Il saisit l'occasion pour donner des informations concernant les projets en cours et les thèmes d'actualité. Les modifications structurelles aussi bien dans le domaine hospitalier que dans le secteur ambulatoire continuent d'impacter les ressources au détriment de la formation postgraduée. Il est donc important que ceux qui en ont la responsabilité s'engagent davantage. Dans les hôpitaux, la valeur de l'enseignement et de la formation sont concurrencées par la valeur des services et les enjeux économiques. L'ISFM essaie de renforcer et de motiver les formateurs à développer de nouveaux projets et de nouvelles initiatives. Mais ces activités ont un coût qui se reflète dans le budget et les comptes annuels. Les comptes annuels
2014 ne traduisent donc pas une mauvaise gestion mais l'expression d'une responsabilité affirmée et déterminée pour l'avenir de la formation postgraduée et continue. Pour qu'une institution continue d'améliorer la qualité, elle doit investir dans la formation postgraduée. Actuellement, l'Office fédéral de la santé publique prépare l'accréditation 2018 pour tous les cursus de formation postgraduée. Cette procédure, qui a lieu tous les sept ans, occasionne une charge administrative supplémentaire pour l'ISFM et les sociétés de discipline médicale. Le 23 septembre 2015, le Medical Education Symposium sera organisé pour la deuxième fois à Berne. Il sera dédié aux perspectives de la formation postgraduée. Toute personne intéressée est cordialement invitée à y participer.

\subsection{Rapport de gestion 2014 de l'ISFM}

Christoph Hänggeli/directeur de l'ISFM informe du rapport de gestion 2014 de l'ISFM tiré à 4500 exemplaires et remis entre autres aussi à tous les directeurs des établissements de formation postgraduée. Entre-temps, des étapes importantes ont été franchies pour les trois objets majeurs de l'exercice 2014.

Le 20 mars 2015, les Chambres fédérales ont adopté la révision de la loi sur les professions médicales (LPMéd) Il aura fallu cinq ans d'un difficile travail de persuasion, accompagné d'un nombre impressionnant de séances et de prises de position, pour faire accepter des points de vue importants du corps médical et des patients. La question de savoir si un médecin doit au moins parler une langue nationale a été âprement débattue. Le Conseil national et le Conseil des Etats ne sont parvenus à une solution satisfaisante qu'à la conférence finale de conciliation. L'exigence de créer un registre complet des professions médicales a été encore plus importante que la compétence linguistique. Grâce à elle, il sera désormais possible de vérifier officiellement tous les diplômes étrangers avant qu'un médecin ne commence à exercer en milieu hospitalier. Le Conseil fédéral va maintenant édicter les dispositions d'exécution dont l'entrée en vigueur est prévue pour 2016.

En novembre 2014, la Conférence suisse des directrices et directeurs cantonaux de la santé (CDS) a approuvé la Convention intercantonale sur le financement de la formation médicale postgraduée. D'une part, les cantons devront verser aux établissements de formation postgraduée reconnus au minimum CHF 15000 par médecin-assistant pour la formation postgraduée structurée. D’autre part, une compensation intercantonale est prévue entre les cantons qui occupent un grand nombre de médecins-assistants et les cantons qui n'en forment qu'un petit nombre. L'approbation de 18 cantons est nécessaire pour la ratification de cette convention. 


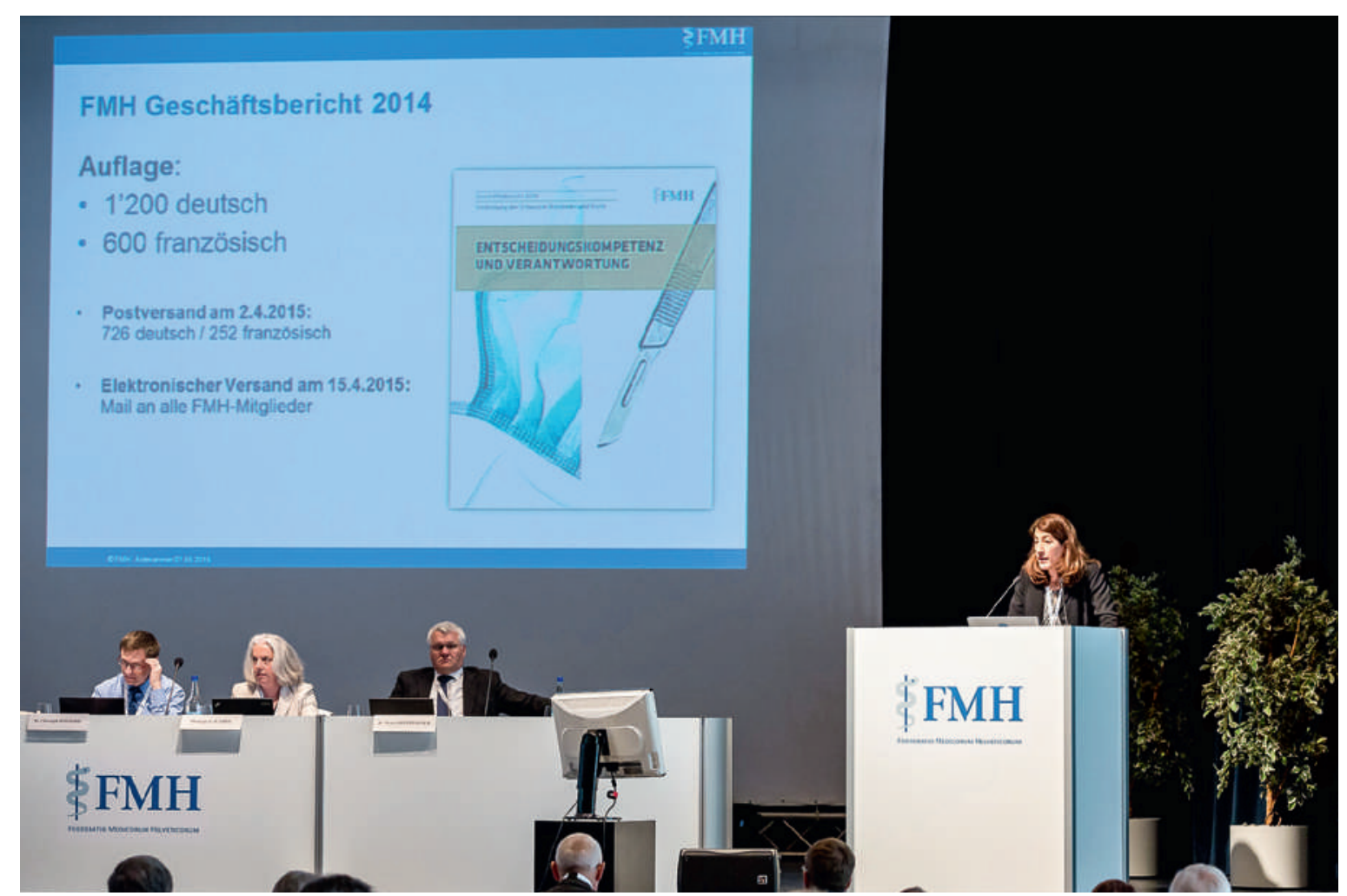

Anne-Geneviève Bütikofer, secrétaire générale de la FMH, présente le rapport annuel 2014, qui est publié sous forme imprimée et électronique.

Le logbook électronique comprend actuellement 40 titres de spécialiste et 16 formations approfondies. Il permet de documenter $96 \%$ de toutes les périodes de formation postgraduée accomplies: sur les 10000 médecins-assistants, 7500 sont enregistrés comme utilisateurs. L'objectif est de l'étendre à toutes les disciplines d'ici à l'été 2015. Le logbook électronique permet aussi de gérer directement le cockpit (tableau de bord) et les demandes de titre par le secrétariat de l'ISFM sans que les données ne soient transférées vers un deuxième système.

La version électronique du rapport de l'ISFM peut être consultée sur www.siwf.ch.

\section{Proposition:}

La direction de l'ISFM demande à la Chambre médicale suisse d'approuver le rapport de gestion 2014 de l'ISFM.

\section{Décision:}

Le rapport de gestion 2014 de l'ISFM est approuvé à la majorité.

\subsection{Rapport de gestion 2014 de la FMH}

Anne-Geneviève Bütikofer/SG présente le rapport de gestion 2014 de la FMH avec un nouveau concept visuel. Ce rapport, envoyé en version papier le 2 avril 2015 et par voie électronique le 15 avril 2015, a été consacré au thème central «Compétence décisionnelle et res- ponsabilité» qui a servi de fil rouge et qui a également été au cœur d'une discussion avec la Dresse Yvonne Gilli, conseillère nationale, et le Dr Adrian Wirthner. Il en est ressorti que ces deux notions constituent la clé de voûte d'un grand nombre de sujets aussi actuels qu'importants pour le corps médical. Le rapport de gestion est accompagné de la statistique médicale de la FMH et d'une vue d'ensemble des prestations de la FMH. Ces documents sont également disponibles en ligne. Les rapports du CC, du SG et des divisions décrivent les activités politiques, stratégiques et opérationnelles. Désormais, les mandats internationaux font également l'objet d'un rapport.

Le rapport de gestion de la FMH est tiré à 1200 exemplaires en allemand et 600 en français. D'autres informations peuvent être obtenues sous www.fmh.ch. Jürg Schlup/président FMH remercie la secrétaire générale au nom du CC pour le travail exigeant qu'elle accomplit chaque jour pour la FMH avec une motivation sans faille.

\section{Proposition:}

Le CC de la FMH demande à la ChM d'approuver le rapport de gestion 2014 de la FMH.

\section{Décision:}

Le rapport de gestion 2014 de la FMH est approuvé par 101 voix et 2 abstentions. 


\section{Comptes annuels 2014}

\subsection{Comptes annuels de l'ISFM}

Ces dix dernières années, Christoph Hänggeli/directeur de l'ISFM a présenté chaque fois un résultat positif qui a permis à l'ISFM d'accumuler des réserves à hauteur de CHF 6 millions. Entre-temps, l'ISFM a développé des projets importants pour la formation postgraduée et ainsi réduit les réserves afin de les investir de manière ciblée et judicieuse. De ce fait, les comptes annuels 2014 bouclent avec une perte de CHF 889000 . Cette évolution a déjà été annoncée l'année dernière lors de la présentation des comptes 2013. L'exercice 2013 constitue ainsi un tournant définitif car les charges dépasseront les produits ces prochaines années sans que les conditions-cadres ne changent. L'écart de CHF 287000 par rapport à la perte budgétisée est essentiellement due au logbook électronique qui a occasionné des dépenses nettement plus élevées que prévues en raison de la complexité du projet. D'autres projets et les refacturations internes par la FMH ont également dépassé les sommes budgétisées. Le rapport entre produits et charges est disproportionné en particulier pour les commissions d'opposition et la formation continue. Il est nécessaire d'agir. Les recettes sont générées avant tout par les titres de spécialiste octroyés et dépendent donc de leur nombre. En 2014, l'ISFM a délivré 1500 titres de spécialiste. Il convient de relever que le taux de médecins étrangers qui acquièrent un titre fédéral est désormais de 44\%. Eu égard au budget 2016 et à la planification financière 2017/18, l'ISFM examinera quelles mesures prendre pour rééquilibrer les produits et les charges à long terme.

\subsection{Comptes annuels de la FMH}

Roland Burger/BDO pour ce point à l'ordre du jour. Emanuel Waeber/directeur administratif et financier de la FMH présente les comptes annuels 2014 qui bouclent avec un gain de CHF 1,308 million, suite notamment à la recommandation de la société de révision de dissoudre les provisions latentes à hauteur de CHF 634000 réservées pour la contribution de l'employeur à la caisse de pension PAT BVG. Sans cette dissolution, les comptes auraient été bouclés avec un gain de CHF 674000. Ce résultat positif est également lié à la nonréalisation de projets budgétisés à hauteur de CHF 730000 . Les produits sont cependant plus élevés de CHF 2,54 millions par rapport à l'exercice précédent alors que les charges ont augmenté de CHF 1,93 million. Les produits et les charges doivent continuer de faire l'objet d'un suivi précis et le frein aux dépenses, tel que l'a décidé la ChM du 30 octobre 2014, soit à partir du budget 2016, doit être maintenu.

Le fonds de roulement s'élève à CHF 17,6 millions, ce qui correspond à $73 \%$ de la somme inscrite au bilan. Les dettes à court terme avec un capital étranger sont net-

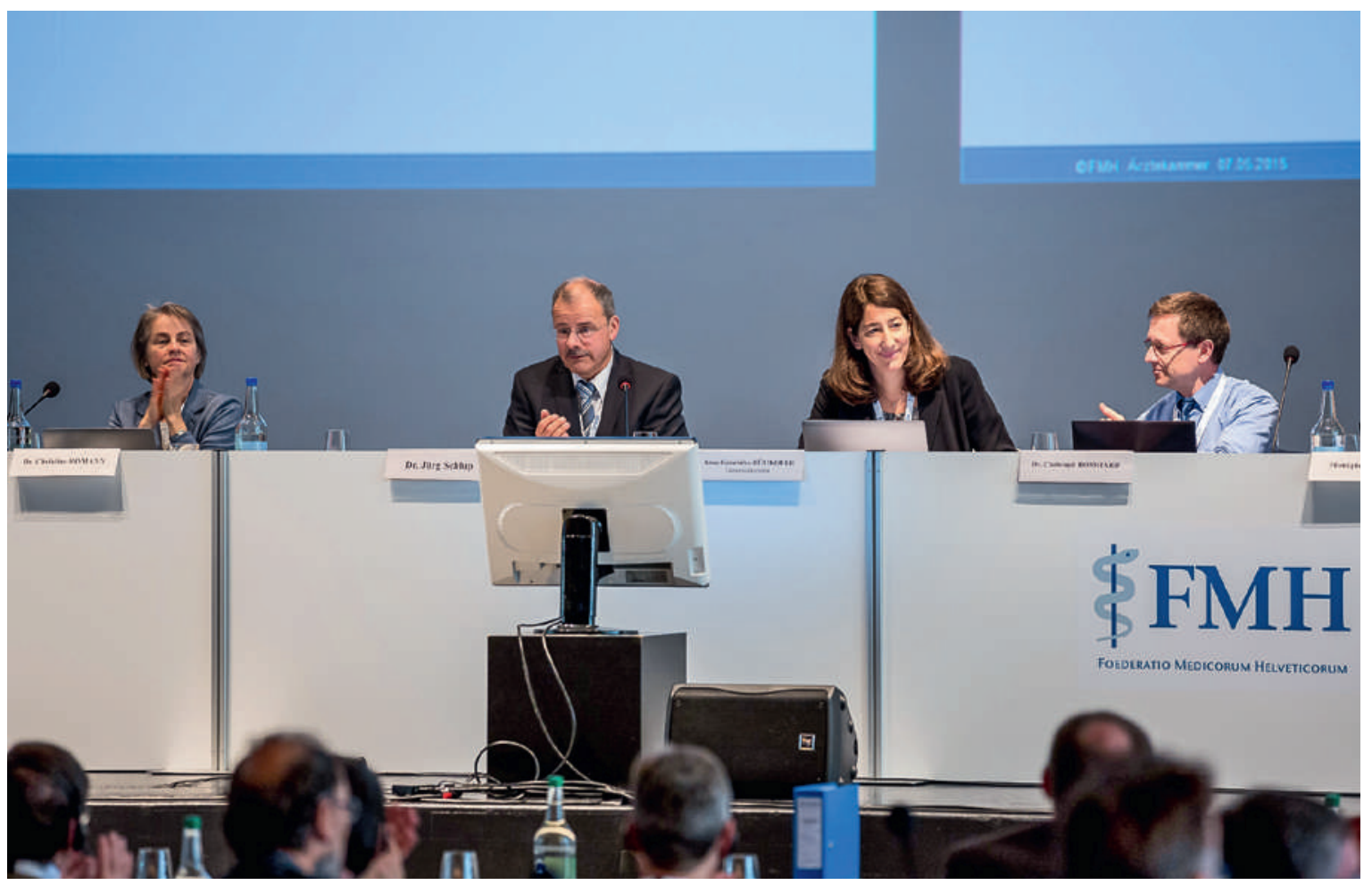

Jürg Schlup ( $2^{\mathrm{e}}$ à partir de la gauche), Anne-Geneviève Bütikofer ( $2^{\mathrm{e}}$ à partir de la droite) et les membres du Comité central dirigent la séance avec entrain et concentration au vu de l'ordre du jour particulièrement chargé. 
tement plus basses qu'en 2013 car les dettes à court terme envers des tiers ont été inférieures de CHF 1,4 million. Le produit d'exploitation dépasse de 2,5 millions celui de l'exercice précédent. La part du capital propre reste de $59 \%$.

Le CC propose de répartir le gain et la perte comme suit: le gain de CHF 1,308 million est attribué au capital de la FMH et la perte de l'ISFM aux réserves de l'ISFM.

\subsection{Rapport de la Commission de gestion}

Adrian Sury/président de la CdG communique les activités de la CdG dont la composition est restée inchangée. L'exercice 2014 a été marqué par l'avenir financier de la FMH, qui deviendra critique d'ici à trois ans en raison de l'écart toujours plus grand entre recettes et dépenses, et du déficit structurel. Dans ce contexte, la CdG a soumis le 30 octobre 2014 une proposition à la ChM visant à élargir la marge de manœuvre financière des activités opérationnelles, laquelle a été approuvée par la ChM avec la décision de créer une Task force. Le 6 juin 2014, la CdG a décidé de supprimer le soutien à la réinsertion professionnelle du CC. Le nouveau règlement n'est cependant valable que pour les nouveaux membres du CC et de l'ISFM. Les membres actuels continuent de dépendre de l'ancien règlement (garantie des droits acquis). La CdG a déjà proposé en 2013 d'intégrer l'IPI dans le département eHealth. La ChM prendra le 29 octobre 2015 des décisions à ce sujet. Concernant la carte HPC, une solution meilleur marché se profile à l'horizon. La CdG a suivi les projets suivants: gestion des risques (nouveau droit comptable en collaboration avec l'organe de révision), règlement du personnel qui a été révisé et introduit en 2015, règlement sur les placements, e-gouvernance et stratégie de communication. Le nouveau règlement sur l'indemnisation des séances et des frais pour les organes et commissions de la FMH est déjà appliqué. Entre-temps, la FMH a également obtenu l'accès aux chiffres des éditions EMH.

Le résultat positif des comptes annuels est dû essentiellement à la non-réalisation de différents projets et à la dissolution des réserves latentes. Les comptes annuels 2014 montrent cependant qu'il existe toujours des problèmes financiers structurels.

Le 11 mars 2015, la CdG a participé à la discussion finale sur les comptes d'exploitation et le bilan, et pris acte du résultat des comptes consolidés et de la tenue correcte de la comptabilité 2014. La CdG souligne le dialogue constructif avec le CC et l'ISFM et leur excellent travail. Elle demande d'approuver les comptes 2014 de la FMH et de l'ISFM et d'en donner décharge.

Roland Burger/BDO présente succinctement le rapport de révision sur les comptes annuels de la FMH, lesquels ont été examinés par l'organe de révision à la fin 2014. Selon son appréciation, les comptes annuels arrêtés au 31 décembre 2014 sont conformes à la loi suisse et aux Statuts de la FMH. Au nom de BDO, il remercie le CC de la confiance témoignée et de la bonne collaboration avec le SG de la FMH.

Comme les délégués présents ne demandent pas la parole ni ne posent de questions sur les comptes annuels de l'ISFM et de la FMH, le président passe au vote.

\subsection{Approbation des comptes annuels de l'ISFM Proposition:}

La direction de l'ISFM propose à la Chambre médicale d'approuver les comptes annuels 2014 de l'ISFM.

Décision:

Les comptes annuels de l'ISFM sont approuvés par 101 voix et 4 abstentions.

\subsection{Approbation des comptes annuels de la FMH}

Proposition:

Le Comité central propose à la Chambre médicale d'approuver les comptes annuels 2014 de la FMH.

\section{Décision:}

Les comptes annuels de la FMH sont approuvés par 101 voix et 7 abstentions.

\section{Octroi des décharges}

Le comité de l'ISFM et le Comité central de la FMH demandent à la Chambre médicale de suivre les recommandations de la CdG et de l'organe de contrôle et de leur donner décharge pour l'exercice 2014.

\subsection{Octroi de la décharge à l'ISFM}

Proposition:

Le comité de l'ISFM demande à la Chambre médicale de suivre les recommandations de l'organe de contrôle et de la CdG et de donner décharge au comité de l'ISFM pour l'exercice 2014.

\section{Décision:}

La Chambre médicale donne décharge au comité de l'ISFM à l'unanimité pour l'exercice 2014.

4.2 Octroi de la décharge au Comité central de la FMH Proposition:

Le Comité central de la FMH demande à la Chambre médicale de suivre les recommandations de l'organe de contrôle et de la CdG et de donner décharge aux membres du Comité central pour l'exercice 2014.

Décision:

La ChM donne décharge au Comité central de la FMH à l'unanimité pour l'exercice 2014. 


\section{Modifications des Statuts, du Règlement d'exécution et du Code de déontologie}

\subsection{Modification des Statuts de la FMH}

\subsubsection{Proposition de la reconnaissance de} l'association des médecins de famille et de l'enfance en tant qu'organisation autorisée à intervenir (selon annexe III)

Cette proposition a entre-temps été retirée par l'Association des médecins de famille et de l'enfance.

\subsubsection{Demande de reconnaissance des sociétés} suisses de chirurgie vasculaire et de chirurgie thoracique, et attribution d'un siège respectif à la Chambre médicale (selon annexe II) Les deux formations approfondies en chirurgie vasculaire et en chirurgie thoracique sont devenues début 2015 des titres de spécialiste que la Chambre médicale doit aujourd'hui reconnaître formellement. Werner Bauer/président de l'ISFM explique qu'en vertu des art. 18 et 33 des Statuts de la FMH, les sociétés de discipline médicale ont droit à un siège au sein de la ChM. 58 sièges leur sont réservés.

\section{Proposition:}

Reconnaissance des deux nouvelles sociétés de discipline médicale par la ChM et attribution d'un siège respectif à la ChM.

\section{Décision:}

La proposition est adoptée par 136 voix contre 2 .

\subsection{Modification du Règlement d'exécution de la FMH}

\subsubsection{Délais de recours en cas d'exclusion conformément à l'art. 9, al. 4 et 5 des Statuts; nouvel art. 3 bis}

A l'occasion du traitement d'un recours contre l'exclusion d'un membre d'une organisation de base, il est apparu que, ni les Statuts, ni les règlements de la FMH ne prévoyaient de délai spécifique pour recourir contre une décision d'exclusion d'un membre (à l'exception des procédures de déontologie). Le CC souhaite combler cette lacune et demande à la $\mathrm{ChM}$ de soutenir sa proposition d'introduire un délai de recours de 30 jours dans le RE.

Andreas Haefeli/AG demande si la réglementation proposée tient compte des vacances judiciaires.

Hanspeter Kuhn/chef de la division juridique de la FMH précise que ces questions sont réglées dans le droit civil et qu'il n'est donc pas nécessaire de les reprendre ici en détail.

Hans-Jakob Rietmann/AGZ s'interroge si le délai de 30 jours commence à courir à partir de l'envoi du recours ou de sa réception par le membre.

Hanspeter Kuhn/FMH explique que le délai de recours commence à la réception de la notification.

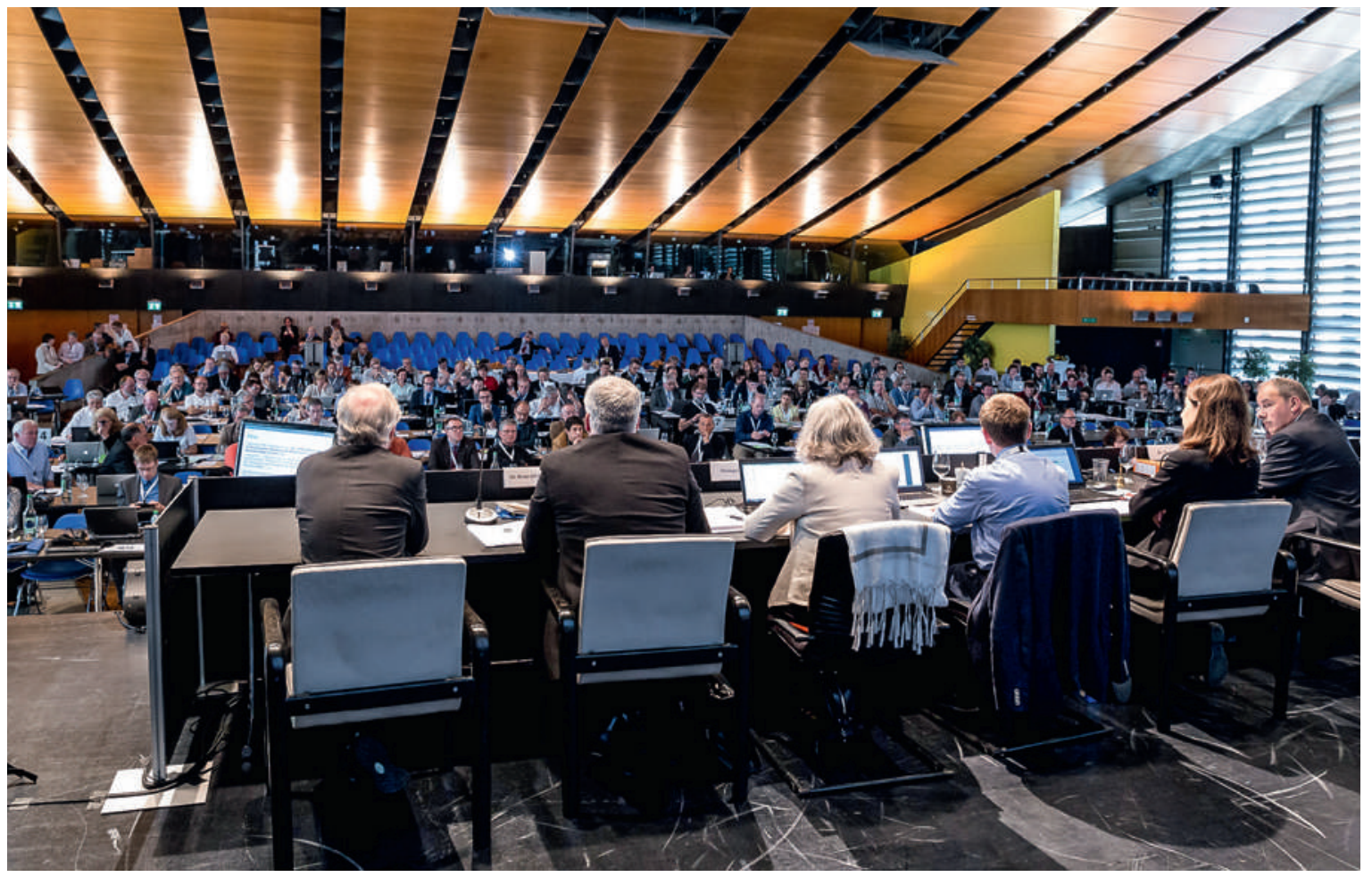

Vue de la salle depuis le podium où siège le Comité central de la FMH. 
Proposition:

Modification du RE par l'introduction de la disposition suivante:

Art. 3bis Délai de recours et indication des voies de droit en cas d'exclusion selon l'art. 9, al. 4 et 5 des Statuts. Le délai de recours est de $\mathbf{3 0}$ jours dès la notification. La décision indiquera la possibilité de recourir dans les 30 jours auprès du CC (en cas d'exclusion selon l'art. 9 al. 4 des Statuts) respectivement, auprès de la ChM (en cas d'exclusion selon l'art. 9 al. 5 des Statuts).

Décision:

La proposition est adoptée par 162 voix, sans opposition ni abstention.

\subsection{Adaptation du Code de déontologie de la FMH}

\subsubsection{Modification de l'art. 44}

Me Anne-Sylvie Thiébaud/Division juridique de la FMH et Me Michel Meier/AeG SO sont accueillis pour traiter ce point. Anne-Sylvie Thiébaud/FMH souligne en introduction qu'après les différentes rencontres avec les commissions de déontologie cantonales en 2013, la grande majorité d'entre elles a plébiscité un modèle de règlement unifié ainsi que la mise en place d'une banque de données des décisions. Début 2014, le CC a alors chargé un groupe de travail de proposer des modifications dans le règlement de la Commission de déontologie de la FMH et de mettre en place une banque de données des décisions rendues. Le groupe de travail, dirigé par le président de la FMH et composé du Dr R. Raggenbass/président de la Commission de déontologie du canton du Valais, de la Dresse M. Winterhalter/ancienne présidente de la Commission de déontologie de Bâle-Ville, du Dr U. Strebel/ancien vice-président de la Commission de déontologie de la FMH, de Me Michel Meier/ avocat de la Société de médecine du canton de Soleure et de Me Anne-Sylvie Thiébaud/avocate responsable du domaine de la déontologie à la FMH, s'est saisi de cette question et a demandé au CC de modifier l'art. 44 du Code de déontologie.

Michel Meier explique que la modification de l'art. 44 du Code de déontologie de la FMH (CoD) permettrait, en cas de lacune, une application subsidiaire des dispositions du Code de procédure civile (CPC) et non plus celles de la loi fédérale sur la procédure administrative (PA). La procédure civile est celle qui se rapproche le plus de la procédure appliquée devant les commissions de déontologie. Ces dernières ont un statut particulier puisqu'elles ne sont ni des autorités pénales, ni des autorités civiles ni même administratives. Elles prononcent toutefois des sanctions disciplinaires. Si l'art. $44 \mathrm{CoD}$ renvoie actuellement aux règles de la procédure admi- nistrative, ce renvoi est lié à des raisons historiques car pendant longtemps, la procédure civile n'était pas unifiée en Suisse. Elle l'est depuis 2011 avec l'entrée en vigueur du CPC. La révision de l'art. 44 permettra aux organisations de base de ne plus devoir se doter d'une réglementation pour autant que le code de déontologie de la FMH soit directement applicable.

Le CC tout comme le groupe de travail et la plupart des organisations de base auxquelles le modèle de règlement de procédure a été soumis, trouvent ce renvoi approprié. L'application du CPC n'intervient de plus que lorsqu'une question n'a pas été réglée dans le Code de déontologie ou dans le règlement de procédure. Le CC propose à la ChM la modification de l'art. 44 du Code de déontologie de la FMH qui permettrait, en cas de lacune, une application subsidiaire des dispositions du CPC et non plus celles de la loi fédérale sur la procédure administrative (PA).

L'AD soutient cette proposition à l'unanimité. Si elle est votée, cette modification entrerait en vigueur au terme du délai d'opposition du présent procès-verbal. La mise en ligne d'une banque de données est prévue au $1^{\text {er }}$ janvier 2016.

Jürg Schlup/président FMH remercie toutes les sociétés cantonales de médecine, Anne-Sylvie Thiébaud et Michel Meier de l'énorme travail fourni.

\section{Proposition:}

La ChM valide l'adaptation suivante du Code de déontologie de la FMH:

Art. 44 (droit de procédure applicable)

Si une question déterminée ne peut trouver réponse ni dans le Code de déontologie, ni dans les règlements de la société cantonale de médecine, de l'ASMAC, de l'AMDHS ou de la Commission de déontologie de la FMH, les dispositions du Code de procédure civile (CPC) sont applicables.

Décision:

La proposition est adoptée par 149 voix, sans opposition ni abstention.

\subsubsection{Révision de l'art. 18: reprise des directives} de l'ASSM concernant la "Distinction entre thérapie standard et thérapie expérimentale dans le cadre individuel»

En mai 2014, l'ASSM a approuvé les nouvelles directives et recommandations médico-éthiques «Distinction entre thérapie standard et thérapie expérimentale dans le cadre individuel». La Chambre médicale a refusé de reprendre ces directives dans le Code de déontologie de la FMH lors de sa séance du 30 octobre 2014 suite à deux interventions. Sur la base des remarques de la Chambre médicale d'octobre 2014, des entretiens ont eu lieu entre la FMH et l'ASSM. Les passages contes- 
tés des directives ont été révisés. Les critiques ne portaient pas sur les directives proprement dites, mais sur les recommandations.

Le Prof. Christian Kind/président de la Commission d'éthique de l'ASSM présente les directives révisées. La nouvelle version a pour objectif de préserver la liberté thérapeutique dans les cas individuels complexes, de prévenir les abus et de distinguer entre thérapie standard et thérapie expérimentale. Elle comble le vide laissé délibérément par le législateur qui envisage la possibilité d'une autorégulation des institutions médicales, comme l'avait déjà évoqué la solution mentionnée dans la réponse du Conseil fédéral du 23 février 2011 à la motion 11.3001 - Essais thérapeutiques. Les malentendus se sont principalement concentrés sur la question de la définition de la thérapie standard. Les standards peuvent être définis à des niveaux différents et poursuivre des objectifs différents. Les directives de l'ASSM se concentrent exclusivement sur des recommandations spécialisées et visent le devoir de diligence du médecin dans le cadre individuel. Elles n'incluent en aucune façon une application directe à d'autres niveaux avec d'autres objectifs.

Lors de la recherche d'un consensus, les parties se sont mises d'accord pour déclarer que la présence d'une personne de confiance était obligatoire lors de l'information au patient avant une thérapie expérimentale. Le tableau représentant les différents niveaux de standardisation a été supprimé des directives. Enfin, ces malentendus ont incité l'ASSM de publier séparément ses recommandations et ses directives.

Le CC demande à la ChM de reprendre la nouvelle version des directives dans le Code de déontologie de la FMH. La reprise de ces directives dans le Code de déontologie de la FMH doit d'une part donner des instructions claires aux médecins et d'autre part sécuriser les patients. La décision de la ChM a lieu sous réserve de l'acceptation de la révision des directives par le Sénat de l'ASSM qui siègera le 19 mai 2015.

Charles-A. Favrod-Coune/VD et membre de la Commission de déontologie de la FMH souligne l'importance des directives dans le Code de déontologie. Il a constaté le matin même que l'ASSM a encore publié l'ancienne version. Selon lui, deux versions différentes ne peuvent coexister. Il faut s'assurer que l'ASSM publie ses directives sans recommandations et sans aucune référence à ces dernières. Il demande par ailleurs une publication simultanée de ces directives par l'ASSM et la FMH. Il rappelle enfin que le compendium n'est plus complet et ajoute qu'il souhaiterait que la traduction française soit un peu meilleure et respecte les règles épicènes. S'agissant de la publication simultanée, Hanspeter Kuhn/ $F M H$ souligne que l'ASSM pourra mettre en vigueur les directives dès la décision du Sénat qui siègera le 19 mai 2015. A la FMH, il faudra attendre le délai d'opposition de 60 jours suivant la publication du procès-verbal. Le Prof. Christian Kind/ASSM a conscience des questions de genre, mais souhaite conserver la version française existante. L'ASSM n'utilise généralement qu'un seul genre en mentionnant dans une note de bas de page que l'autre genre est inclus. Concernant le compendium, il propose à Charles-A. Favrod-Coune une clarification de ce point de manière bilatérale.

Les deux opposants de la ChM du 30 octobre 2014, Jürg Nadig et Beat Gafner, approuvent la version remaniée et remercient d'avoir pris en compte leurs critiques. Jürg Nadig fait remarquer que sa société de discipline médicale sera ici particulièrement mise à l'épreuve. Il est prévu de publier un article dans le BMS qui définira ce que la société de discipline médicale entend par thérapie standard afin de pouvoir effectuer la distinction avec la thérapie expérimentale.

\section{Proposition:}

Adaptation du Code de déontologie de la FMH: Art. 18 Directives de l'Académie suisse des sciences médicales (complément)

Les directives de l'Académie suisse des sciences médicales sont applicables dans les domaines suivants: [...] Distinction entre thérapie standard et thérapie expérimentale dans le cadre individuel. [...]

\section{Décision:}

La proposition est adoptée par 158 voix et 1 abstention.

La décision de la ChM a lieu sous réserve de l'acceptation de la révision des directives par le Sénat de l'ASSM qui siègera le 19 mai 2015.

\subsubsection{Actualisation de l'art. 18: reprise des} directives révisées "Exercice de la médecine auprès de personnes détenues»

Selon Monique Gauthey/CC FMH, l'exercice de la médecine notamment lors du rapatriement de personnes faisant l'objet d'un renvoi de Suisse était, et reste délicat, et ne fait pas l'objet d'une réglementation adaptée à la pratique. C'est ce qui a incité la Conférence des directrices et directeurs des départements cantonaux de justice et police (CCDJP) à proposer le 15 mai 2013 à la conseillère fédérale Sommaruga que la Confédération et les cantons dialoguent avec le corps médical, en association avec la Commission nationale de prévention de la torture (CNPT). Ce dialogue avait notamment comme objectifs d'améliorer la transparence de l'accompagnement médical des personnes faisant l'objet d'un renvoi, en vue d'un soutien plus large au sein du corps médical, d'augmenter la confiance du corps médical concernant l'accompagnement médical et d'aborder 


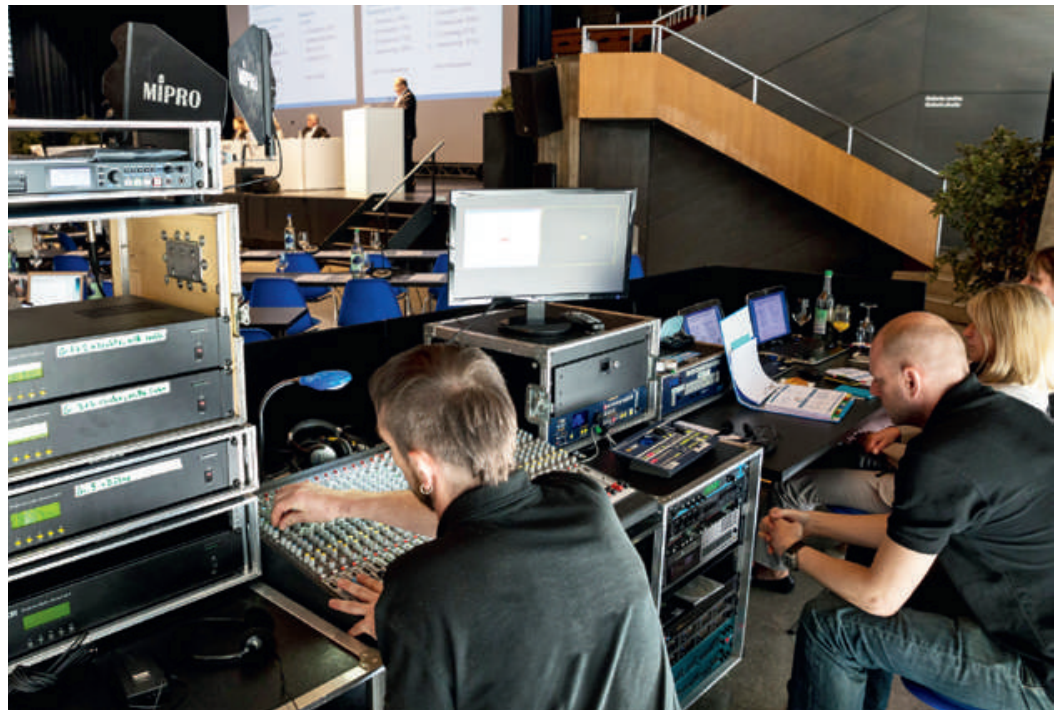

Les techniciens veillent en coulisse au bon déroulement de la séance.

la question du flux de données médicales avant le renvoi. En complément des discussions qui se sont tenues depuis 2013 entre le Secrétariat d'Etat aux migrations, l'ASSM et la FMH sur le rôle des médecins en lien avec les rapatriements sous contrainte de requérants d'asile refoulés, l'ASSM a révisé les directives «Exercice de la médecine auprès de personnes détenues» après avoir analysé cette situation complexe et clarifié les points de divergence ainsi que les différents rôles. Ces directives décrivent les missions du corps médical telles que cette nouvelle répartition des rôles les définit. Cette nouvelle répartition des rôles doit encore faire l'objet d'une évaluation dans un délai de quelques mois, afin de voir si la mesure permet d'obtenir le résultat escompté sur la réduction des risques médicaux.

Le Prof. Christian Kind/ASSM présente la nouvelle réglementation, à caractère contraignant pour tous les médecins. Une nouvelle annexe aux directives existantes ainsi que la publication d'une liste de contre-indications médicales au vol de rapatriement devraient permettre de clarifier le rôle des médecins. Le certificat d'aptitude au vol ("fit to fly») ne sera plus délivré à l'avenir par le médecin traitant, mais par le médecin accompagnateur. C'est lui qui décidera en dernière instance de l'aptitude au vol. Le Secrétariat d'Etat aux migrations a signé une convention dans ce sens avec la société Oseara chargée d'accompagner les vols.

Thomas Heuberger/BE demande si la question du traitement forcé pendant le vol est clarifiée et si la décision incombe le cas échéant au pilote.

Prof. Christian Kind/ASSM répond que les directives mentionnent la question du traitement forcé. Un traitement forcé n'est possible qu'aux mêmes conditions que pour les personnes non détenues. A savoir, une incapacité de discernement et l'absence de toute alternative pour aider le patient. Les directives sur le traitement forcé sont en cours d'élaboration et seront soumises aux délégués à la prochaine ChM. Selon lui, le pilote est en droit de décider de ce qu'il advient du passager, mais pas sur le plan médical. La décision du traitement revient au médecin accompagnateur.

Thomas Heuberger/BE s'étonne de cette distinction car la responsabilité du vol et des passagers incombe en fin de compte au pilote. En cas d'incidents susceptibles de compromettre la sécurité du vol, l'avis du pilote est primordial.

Le Prof. Christian Kind/ASSM précise que le pilote peut exiger du médecin que le patient ne compromette pas la sécurité du vol. La manière d'y parvenir relève de la responsabilité du médecin.

Daniel Jud/TG souhaite en savoir plus sur la société Oseara.

Le Prof. Christian Kind/ASSM ne dispose d'aucune information supplémentaire et renvoie au site internet. La société a été créée spécialement dans ce but. Le médecin adjoint, le Dr Adrian Businger, était également présent lors des discussions.

Le Prof. Jean-Michel Gaspoz/SSMI, chef du Département de médecine communautaire, de premier recours et des urgences aux HUG, dont dépend également la médecine pénitentiaire, salue les présentes directives qui clarifient les rôles des différents acteurs. Il lui tient à cœur que le médecin traitant connaisse son rôle et l'assume sans être contraint de délivrer un certificat d'aptitude au vol ni d'être instrumentalisé par les autorités politiques et policières. Le rôle du médecin traitant consiste à déterminer l'état de santé du patient et à transmettre ces données, sous réserve de l'accord de la personne concernée, au médecin chargé de l'évaluation de l'aptitude au vol. Mais il n'est pas responsable des conséquences d'une ordonnance administrative ou de police.

Proposition:

Le CC demande à la ChM d'actualiser le renvoi aux directives de l'ASSM «Exercice de la médecine auprès de personnes détenues»: en référence à la directive révisée.

\section{Décision:}

La proposition est adoptée par 152 voix contre 1. La décision de la ChM a lieu sous réserve de l'acceptation de la révision des directives par le Sénat de l'ASSM qui siègera le 19 mai 2015.

\section{Elections, élections de confirmation, élections de remplacement}

\section{1 Confirmation des délégués nommés à l'AD}


6.2 Confirmation des suppléants nommés à l'AD Contrairement aux annonces qui avaient été faites concernant les points 6.1 et 6.2, aucune demande n'a été finalement soumise. Les élections de confirmation ne sont donc plus nécessaires.

Jürg Schlup/président FMH saisit l'occasion pour informer les délégués des raisons pour lesquelles le CC n'a pas demandé d'élections pour remplacer Ernst Gähler. D'une part, le délai ordinaire pour soumettre une candidature a expiré deux jours après les obsèques de Ernst. D'autre part, la Task force de la ChM propose aujourd'hui une réduction du CC. Le CC attendra donc la décision qui sera prise aujourd'hui par la ChM avant de planifier l'élection d'un remplaçant à la prochaine ChM.

\section{Planification financière / Contrôle financier}

\subsection{Contrats de prestations de la FMH}

Anne-Geneviève Bütikofer/SG FMH expose les raisons qui ont incité le CC en 2013, dans le cadre de la stabilisation financière, à conclure un contrat de prestations avec tous les bénéficiaires d'un soutien financier annuel de plus de 10000 francs de la part de la FMH. Une procédure a été présentée aux délégués de la $\mathrm{ChM}$ du 3 octobre 2013. Quelques partenaires ont cependant remis en question la base de décision sur laquelle se fondaient les contrats de prestations. Le 8 mai 2014, la ChM a pris connaissance des nouveaux contrats et de l'engagement entre la FMH et les bénéficiaires de soutiens financiers. La plupart des partenaires ont accepté le projet de contrat qui leur a été soumis, quelques-uns ont toutefois refusé le contrat et se sont appuyés sur une décision antérieure de la ChM en faisant valoir qu'elle constituait une base légale suffisante. Faute de temps, la confirmation formelle prévue pour la ChM du 30 octobre 2014 a dû être reportée à la ChM de ce jour.

Fiorenzo Caranzano/TI n'a rien contre le principe des contrats de prestations. Il souhaite néanmoins qu'ils correspondent à la situation réelle.

Anne-Geneviève Bütikofer/SG FMH remercie de cette précision et informe que le Secrétariat général de la FMH a entre-temps obtenu des organisations concernées l'assurance qu'elles concluront les contrats de prestations exigés. Un débat constructif a permis de clarifier les questions parfois complexes et très hétérogènes, notamment concernant les maisons d'édition. Le contrat de prestations vise à régler la relation et les modalités entre le fournisseur et l'acheteur d'une prestation, et sera contrôlé par la division Administration et finances. La procédure correspond à celle déjà présentée auparavant.

\section{Proposition:}

La ChM décide que la FMH doit subordonner tout soutien financier de plus de CHF 10000 par année à la conclusion d'un contrat de prestations. Ce dernier est signé ou renouvelé entre la FMH et le bénéficiaire de la prestation avant tout versement.

\section{Décision:}

La proposition est adoptée par 152 voix et 4 abstentions.

\section{Stabilisation budgétaire de la FMH}

Jürg Schlup/Président FMH signale que l'entrée en matière sur ce point est litigieuse, compte tenu de la proposition de non-entrée en matière déposée par l'ASMAC et de la proposition de renvoi déposée par la SMSR. Au vu de ces propositions, il convient de procéder d'abord à des débats sur l'entrée en matière, puis de voter sur ces deux propositions. Un examen détaillé suivra. Notre Service juridique a sollicité un avis de droit auprès du Prof. H. M. Riemer sur le déroulement proposé. Les débats relatifs à l'entrée en matière se dérouleront de la manière suivante: la Task force interviendra d'abord à titre introductif, puis la CdG interviendra à titre complémentaire, nous passerons ensuite à la proposition de l'ASMAC, à celle de la SMSR, aux interventions du CC et du Service juridique de la FMH, aux débats en plénum sur l'entrée en matière, et enfin à l'intervention finale de la Task force. Il sera ensuite procédé au vote sur la non-entrée en matière ou le renvoi.

\subsection{Débats relatifs à l'entrée en matière}

Josef Widler/AGZ propose de limiter le temps de parole à deux minutes dans le cadre des débats relatifs à l'entrée en matière.

Motion d'ordre Josef Widler :

Le temps de parole est limité à deux minutes dans le cadre des débats relatifs à l'entrée en matière.

Décision :

La motion est acceptée par 120 voix contre 10 et 6 abstentions.

Felix Kurth/ASMAC considère qu'il s'agit de deux propositions différentes. L'ASMAC demande la non-entrée en matière et l'absence de toute présentation, la SMSR souhaite d'abord entendre la Task force, puis renvoyer le rapport. On ne peut pas revoter ultérieurement sur l'opportunité de procéder ou non à la présentation.

Daniel Schröpfer/ASMAC précise que la présentation est prévue dans la proposition de la SMSR. On peut donc se demander pourquoi il faudrait procéder maintenant déjà à un vote sur l'opportunité ou l'inopportunité de la présentation. 
Hanspeter Kuhn/FMH recommande, comme prévu, de procéder à des interventions brèves et de ne pas écouter la présentation dans son intégralité. C'est aussi ce que recommande le Prof. Riemer. L'avis de droit du Prof. H.M. Riemer conclut qu'il faut annoncer les points soumis au vote dans les règles de l'art. Pour ce faire, des documents contenant des informations exhaustives sont nécessaires, afin qu'il soit possible de préparer le vote comme il convient. Selon le Prof. H.M. Riemer, tel n'a pas été le cas en l'espèce, et cette obligation n'a pas été respectée en ce qui concerne deux des points. La Task force n'a fourni aux délégués qu'un bref document, et non l'analyse complète. Par ailleurs, les délégués n'ont pas non plus reçu le procès-verbal de la discussion de l'AD sur ce sujet.

Beat Gafner/BE propose de procéder d'abord à la présentation de KPMG.

Proposition Gafner:

Il convient de procéder d'abord à la présentation de KPMG. Seule cette présentation permettra de délibérer et de déterminer la suite de la procédure. Décision:

La proposition est rejetée par 91 voix contre 41 et 11 abstentions.

Après le rejet de la proposition Gafner, la SMCB fait une autre proposition. Elle souhaite entendre la présentation de KPMG même s'il n'est pas procédé à un examen détaillé.

\section{Proposition SMCB:}

Il convient d'entendre la présentation même s'il n'est pas procédé à un examen détaillé.

Décision:

La proposition est rejetée par 83 voix contre 39 et 7 abstentions.

\subsubsection{Intervention préliminaire de la Task force}

Peter Wiedersheim/Task force cite un extrait du procèsverbal de la ChM du 30 octobre 2014. Il ressort de cet extrait que 95\% des recettes sont réservées aux dépenses liées. Cela ne laisse donc aucune marge de manœuvre pour les nouvelles tâches urgentes et cela génère une divergence entre les recettes et les dépenses. Le 30 octobre 2014, la ChM a constitué une Task force à laquelle elle a assigné les objectifs suivants: équilibrer durablement les dépenses et les recettes, aménager une liberté d'action d'au moins $20 \%$ pour les projets prioritaires, réduire les tâches accessoires, et élaborer de nouvelles possibilités de financement.

Lors de sa séance du 26 novembre 2014, l'AD a élu un secrétaire, Daniel Trachsel, puis les membres suivants à une nette majorité: Adrian Sury/CdG, Alexander Zimmer/CdG, Brigitte Muff/AD, Pierre Vallon/AD, Peter Wiedersheim/AD. Brigitte Muff ayant décliné cette nomination, c'est Karin Stadlin qui a été élue à sa place. La présidence de la Task force a été confiée à l'économiste externe, le Dr Walter Gratzer/KPMG. Emanuel Waeber, chef de la division Administration et finances du SG de la FMH, a été convié en qualité d'invité. Peter Wiedersheim rappelle aux délégués que c'est la ChM qui a confié le mandat à la Task force. Celle-ci, en sa qualité de mandataire, est tenue de rendre des comptes et d'informer la ChM. Il satisfait à cette obligation en livrant les informations qu'il fournit aujourd'hui. Il est évident pour la Task force qu'il n'est possible d'aménager une liberté d'action de $20 \%$, correspondant à une somme allant de 4 à 6 millions de francs environ, qu'en réduisant les coûts dans un premier temps, puis en augmentant les cotisations des membres dans un second temps. Peter Wiedersheim précise que la Task force ne souhaite pas clouer la FMH au sol, mais aspire au contraire à une FMH forte dont l'aptitude à voler est optimale. L'état actuel des finances ne donne toutefois pas de signes de force. De plus en plus, la gestion se réduit essentiellement à l'administration. Or, pour gérer l'organisation, le président a besoin de ressources suffisantes. Les tâches de la FMH sont nombreuses et vont en augmentant. La proposition de l'ASMAC ne résout pas cette problématique et affaiblit au contraire la fédération. La Task force recommande donc de rejeter la proposition de l'ASMAC. Seule une telle solution lui permet d'honorer son devoir d'information lors de la ChM de ce jour. Peter Wiedersheim demande de pouvoir présenter au moins les conclusions de la Task force.

\subsubsection{Intervention complémentaire de la CdG} Adrian Sury/CdG renonce à intervenir, car il s'est entretenu avec Peter Wiedersheim au sujet de l'intervention préliminaire de la Task force.

\subsubsection{Proposition de l'ASMAC: non-entrée en} matière sur les propositions de stabilisation budgétaire de la Task force et nouveau mandat confié au CC de la FMH

Daniel Schröpfer/ASMAC remercie KPMG et la Task force pour le travail de qualité qu'ils ont fourni. Il souligne que cela fait déjà plusieurs années que l'ASMAC rend la FMH attentive aux économies potentielles et l'incite à porter un regard critique sur les processus actuels et à reconsidérer l'affectation de ses dépenses. L'ASMAC soutient les efforts d'économie de la FMH depuis 2007 et ne cherche en aucun cas à entraver le travail de celleci par le biais de sa proposition, ni à la déstabiliser, comme P. Wiedersheim vient de le suggérer. C'est au contraire la proposition de la Task force qui fragilise la FMH. En effet, elle ne permet pas de réaliser des écono- 
mies. Par ailleurs, l'ASMAC désapprouve la manière de procéder après la clôture des travaux. Ni le CC, ni le Secrétariat général n'ont été impliqués dans la mise en œuvre des mesures. Il s'agit aujourd'hui de se prononcer sur une série de mesures d'économie sans savoir sur quels postes elles portent concrètement. C'est pourquoi l'ASMAC demande que le rapport de la Task force soit transmis dans son intégralité et en temps voulu aux délégués de la ChM et que les acteurs essentiels participent à la mise en œuvre. Sur la base d'un nouvel état des lieux, la ChM sera en mesure de se prononcer sur des mesures concrètes en octobre 2015.

\subsubsection{Proposition de la SMSR: renvoi au CC du rapport de la Task force relatif à la stabilisation budgétaire}

Pierre-Alain Schneider/GE a évalué les possibilités d'économie explorées par la Task force et considère que la ChM doit prendre connaissance du rapport de cette dernière. Comme l'ont déjà exprimé d'autres délégués, la SMSR est choquée par la forme des propositions et des procédures retenues et par l'absence d'analyse préalable en concertation avec le CC et le SG de la FMH. La Task force a rempli son mandat, mais la ChM n'est pas en mesure de se prononcer sur les mesures d'économie proposées sur la base des documents fournis. Le rapport doit d'abord être discuté au sein du CC, puis avec le Secrétariat général de la FMH. Nous avons confiance en ces deux instances. Le rapport de la Task force a été perçu comme quelque chose de déstabilisant et porte à croire que la fédération est en train de sombrer. C'est pourquoi Pierre-Alain

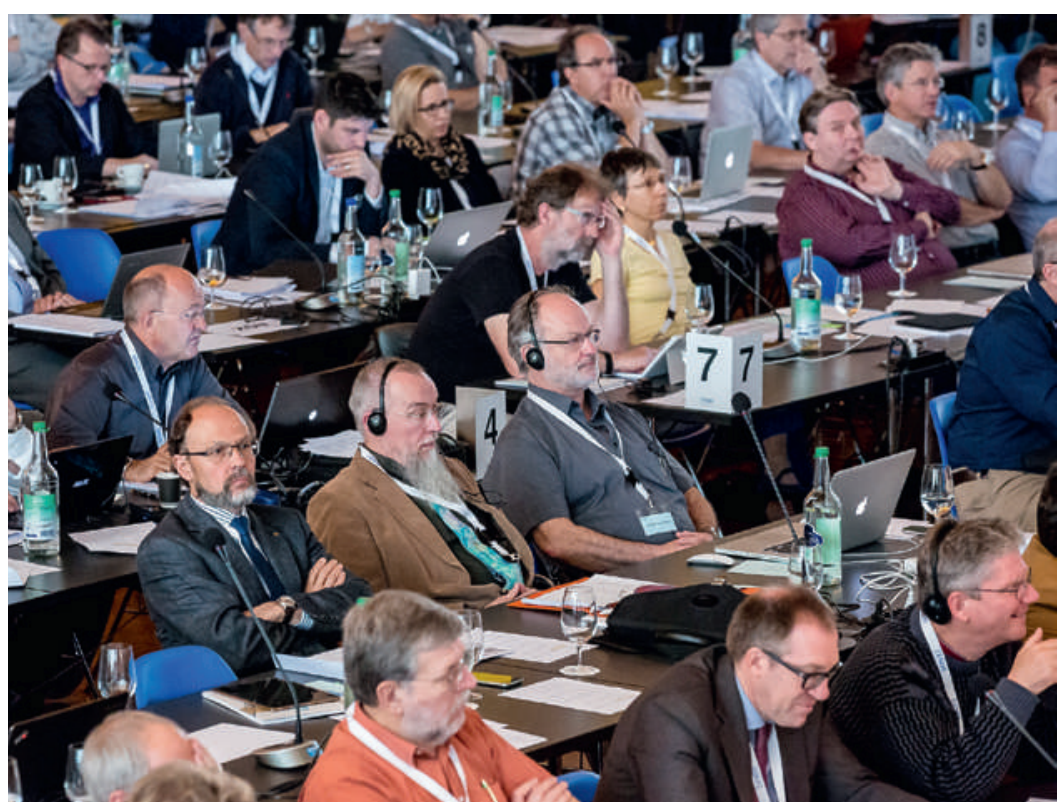

Les délégués à la Chambre médicale suivent attentivement les débats avant de prendre leurs décisions.
Schneider propose que le CC analyse et approfondisse les mesures proposées.

\subsubsection{Recommandations du CC}

Jürg Schlup/président FMH remercie KPMG et la Task force d'avoir assumé la tâche difficile et exigeante d'analyser la FMH et d'établir un rapport sur le sujet. Le CC a conscience du fait qu'ils ont disposé d'un temps très limité pour procéder à cette analyse et rédiger leur rapport compte tenu des contraintes liées à la ChM.

Depuis début 2013, soit depuis deux bonnes années, le CC stabilise le budget de la FMH. A cette fin, il a concrétisé les six mesures suivantes, à savoir trois destinées à améliorer les recettes et trois autres à réduire les dépenses. En vue d'améliorer les recettes, le CC a augmenté les cotisations des membres de la catégorie 3 , a créé la catégorie 10, et redéfini les catégories 7 et 9 . Par ailleurs, en vue de réduire les dépenses, le CC a décidé un frein aux dépenses, soumis les nouvelles commissions à autorisation, et introduit un nouveau règlement relatif aux indemnités de séance qui réduit également les dépenses. En outre, en septembre 2014, le CC a soumis à l'AD une liste des tâches de la FMH en lui demandant de l'examiner et de déterminer lesquelles devaient être maintenues à l'avenir et lesquelles devaient être supprimées. Le CC s'efforce depuis 2013, soit depuis deux ans, de stabiliser progressivement le budget en accroissant les rendements et en réduisant les dépenses. Le CC a également soutenu la proposition de la CdG à la ChM du 30 octobre 2014 parce qu'il souhaite équilibrer le budget de la FMH.

Le 26 novembre 2014, quatre semaines après la dernière ChM, l'AD a fixé la composition de la Task force Stabilisation budgétaire en excluant tant le CC que le SG de ce groupe. Aux yeux du CC, une telle exclusion est incompréhensible et il est incohérent de procéder à un examen stratégique des tâches sans y associer l'organe de direction stratégique de la FMH. En outre, le document de cinq pages présentant les mesures proposées génère incertitude et démotivation au sein du Secrétariat général. Le CC souhaite équilibrer le budget de la FMH. Et les délégués attendent de la FMH des prestations de qualité fournies par des personnes motivées. Il s'agit donc de procéder avec précaution et intelligence. La suppression ou le transfert de postes suscitent énormément d'inquiétude et de gaspillage d'énergie au sein du Secrétariat général. Des mesures irréfléchies risquent de porter un préjudice durable à la FMH. Jürg Schlup souligne une fois encore que le CC s'efforce depuis deux ans d'assainir le budget en accroissant les recettes et en réduisant les dépenses et soumettra un budget équilibré à la ChM l'automne prochain. Après avoir examiné les conclusions du rapport de la Task force, le CC pré- 
sentera à la ChM du 29 octobre 2015 une liste de propositions réalistes sur la base de laquelle les délégués seront en mesure de prendre des décisions claires.

\subsubsection{Service juridique de la FMH}

Hanspeter Kuhn/FMH mentionne qu'il était important de déterminer la marche à suivre exacte, raison pour laquelle le Prof. H.M. Riemer a été contacté. Sous l'angle du droit associatif se pose en outre la question de savoir si les documents fournis aux délégués par la Task force sont suffisants pour pouvoir se prononcer. L'avis de droit pour lequel le Prof. H.M. Riemer a été mandaté conclut que, conformément à l'art. 67, al. 3 CCs, les points nécessitant des décisions doivent être annoncés en bonne et due forme aux sociétaires votants afin qu'ils puissent se préparer en conséquence. Une telle annonce inclut également l'envoi des documents pertinents, ou à tout le moins la communication des informations permettant de les consulter (internet, intranet), car leur examen est indispensable pour se préparer de manière appropriée. L'avis de droit montre qu'en l'espèce, les documents que la Task force a fournis aux délégués ne sont pas suffisants pour se prononcer dans les règles de l'art. L'obligation d'annoncer les points à l'ordre du jour en bonne et due forme a été doublement violée. La convocation du 7 mai 2015 n'était accompagnée que d'un bref résumé de cinq pages. En violation de l'art. 20, al. 3 RE, les délégués de la ChM n'ont pas reçu l'extrait du procès-verbal (chiffre 13) de l'AD du 18 mars 2015 qui leur aurait fourni des éléments d'information supplémentaires en vue de la séance d'aujourd'hui.

Comme cela a déjà été mentionné en guise d'introduction, l'avis de droit du Prof. H.M. Riemer parvient à la conclusion que les points soumis au vote doivent être annoncés en bonne et due forme. A cette fin, des documents contenant des informations exhaustives sont nécessaires pour se préparer et se prononcer. En l'espèce toutefois, cette obligation a été violée à un double titre, comme cela a déjà été dit plus haut.

\subsubsection{Débats en plénum sur l'entrée en matière}

Les personnes suivantes demandent la parole:

Josef Widler/ZH rappelle que la ChM a chargé la Task force d'examiner les mesures envisageables. Ce qui a été fait. Selon lui, il n'est pas nécessaire de décider aujourd'hui s'il convient de mettre en œuvre ces propositions. Il ne partage pas davantage l'opinion du CC selon laquelle il était erroné de ne pas faire siéger le CC au sein du groupe de travail. Il recommande aux délégués d'entrer en matière sur l'examen de détail. L'AGZ soumettra ensuite une contre-proposition qui présentera la marche à suivre jusqu'au vote, pour autant, naturellement, que l'entrée en matière soit acceptée.
Alex von Weymarn/med-swiss.net considère qu'il faut au moins prendre connaissance des travaux de la Task force. Quant à la décision à prendre, c'est une autre question.

Jean-Pierre Pavillon/VD soumet à la réflexion le fait que les décisions prises trop rapidement sont le plus souvent mauvaises. Les bonnes décisions nécessitent du temps. Cela fait déjà sept ou huit ans qu'on peint le diable sur la muraille, mais il n'y a pas de raison de parler à l'heure actuelle d'une situation financière catastrophique de la FMH. Il demande que les délégués prennent connaissance du travail de la Task force. Après analyse de ces propositions, le CC devra soumettre des mesures adaptées et réaliste à la prochaine ChM.

Monique Lehky Hagen/VS conteste les propos de son préopinant. Selon elle, les comptes annuels présentés aujourd'hui ont été embellis par des mesures cosmétiques. Un tel résultat est dû avant tout au fait que des projets n'ont pas été réalisés. Il n'existe pas de réserves, et il n'est pas juste de parler de budget équilibré.

Christian Bernath/FMPP estime que la Task force a rempli son mandat. Il trouve cependant qu'il n'est pas juste de prendre des décisions aujourd'hui déjà, sans savoir quelles en seront les conséquences. La décision de réduire les prestations de services relève de la ChM. Il est possible que la ChM refuse d'entrer en matière sur ce point et décide plutôt d'augmenter les cotisations des membres.

Pour Thomas Heuberger/BE, les propositions de l'ASMAC et de la SMSR vont dans la même direction. La seule différence est que l'ASMAC demande la dissolution de la Task force. Ce qui lui semble important, c'est que ces propositions prévoient toutes deux que l'exécutif puisse assumer sa tâche. En ce sens, il considère qu'il est juste de rejeter la proposition de la Task force, et de débattre par la suite, lors de la prochaine ChM, de propositions viables qui ne bloquent ni le CC ni le Secrétariat général de la FMH. Il plaide en faveur de l'adoption des deux propositions, car elles sont identiques.

Pierre Vallon/Task force demande au Service juridique si seule la consultation du rapport dans son intégralité prémunit la Task force contre un différend juridique relatif à la décision qui pourrait être prise aujourd'hui. Hanspeter Kuhn/FMH interprète la position du Prof. Riemer en ce sens que les informations fournies à ce sujet sont insuffisantes pour pouvoir prendre aujourd'hui des décisions inattaquables. Il existe un risque de contestation, et c'est pourquoi le Prof. Riemer recommande de ne pas prendre aujourd'hui de décisions définitives.

Daniel Ackermann/SSU estime qu'il ne s'agit pas, aujourd'hui, de se prononcer sur les points de détail, mais plutôt de déterminer si on veut ou non entendre les in- 
formations proposées par la Task force. Sur ce point, la proposition de l'ASMAC se distingue très nettement de celle de la SMSR. Leurs conséquences sont différentes. L'année passée, la ChM a confié un mandat et est désormais en droit d'obtenir toutes les informations en la matière. Il incombera au CC et au SG de la FMH d'élaborer les conséquences et la mise en œuvre des mesures ces prochains mois.

Rolf Hunkeler/AGZ se demande pourquoi la Task force devrait être dissoute si l'exécutif est convaincu par ses propositions. Il propose plutôt que tous deux collaborent. En ce sens, il ne soutient pas la proposition de l'ASMAC en ce qui concerne la dissolution de la Task force, mais plutôt celle de la SMSR. Il soutient en principe cette proposition, mais sans dissolution de la Task force.

Jürg Schlup/président FMH répond qu'il s'agirait là d'une nouvelle proposition.

Michaël Hagmann/VD soutient la proposition Hunkeler. S'il est demandé aujourd'hui de ne pas entrer en matière, l'AD doit inviter la Task force à collaborer avec le CC afin de trouver un compromis. La Task force doit être maintenue et des propositions élaborées conjointement devront être présentées à la ChM du 29 octobre 2015.

Note: Rolf Hunkeler/ZH a retiré sa proposition à la fin des débats sur l'entrée en matière.

Hans-Anton Vogel/VEDAG fait observer que la Task force a mené à bien le mandat qui lui a été confié par la ChM. Si les informations demandées sont disponibles, cela permet de discuter des prochaines étapes. Il préconise donc de prendre d'abord connaissance de ces informations, puis d'aller de l'avant.

Hans-Jakob Riedtmann/ZH estime que, si l'instance législative doit voter aujourd'hui sur des points dont l'instance exécutive n'a même pas connaissance, la Task force n'a pas accompli sa tâche et se comporte de manière contraire à la démocratie. Il préconise clairement le renvoi.

Pour Adrian Sury/CdG, en sa qualité de mandante, la ChM doit être informée aujourd'hui en priorité. L'une des raisons de ne pas communiquer à tous les délégués le rapport dans son intégralité relève de la protection des données. En ce qui concerne la prolongation du mandat de la Task force, Alexander Zimmer et lui ne pourront plus assumer cette tâche plus longtemps en raison d'un conflit d'intérêts. En effet, la CdG ne peut pas à la fois assister et contrôler le CC. Il réaffirme que la composition soumise par la CdG à la dernière $\mathrm{ChM}$ prévoyait tant la participation du président de la FMH que celle de la secrétaire générale.

Pierre Vallon/Task force n'accepte pas le reproche de procédure non démocratique. Lors de l'AD du 18 mars
2015, la Task force a présenté un premier rapport au $\mathrm{CC}$ ainsi qu'aux délégués de l'AD. Ceux-ci disposaient déjà d'importantes informations préliminaires. A la demande de la secrétaire générale, les collaborateurs ont aussi été informés. On peut toutefois se demander si un rapport de soixante pages contenant des informations extrêmement sensibles et confidentielles peut et doit sans autre être communiqué à 200 personnes.

Jürg Schlup/président FMH demande que la liste des intervenants soit close par l'intervention du représentant de l'Ordine.

\section{Motion d'ordre Schlup:}

La liste des intervenants est close après l'intervention du représentant de l'Ordine.

\section{Décision:}

La motion d'ordre est acceptée par 101 voix contre 3. Josef Widler/AG considère que la ChM a obtenu ce qu'elle avait demandé. Les droits démocratiques ont été respectés. Selon lui, il est juste que ni le président, ni la secrétaire générale n'aient siégé au sein de la Task force. Il énonce oralement la proposition qui va être déposée: "La ChM prend connaissance des mesures de stabilisation budgétaire prévues par la Task force. Elle considère que les mesures proposées ne réduisent pas les prestations en faveur des membres, et permettent à la FMH d'augmenter sa liberté d'action dans les proportions souhaitées. Elle charge le CC premièrement: d'examiner les mesures proposées. Deuxièmement: de présenter un plan des mesures et un calendrier détaillés à la ChM du 29 octobre 2015. Troisièmement: de préciser, lors de la ChM du 29 octobre 2015, quelles mesures il ne peut ou ne veut pas mettre en œuvre et de motiver son refus. Quatrièmement: de proposer les modifications nécessaires des Statuts, des règlements et des décisions de la ChM. Cinquièmement: d'informer régulièrement l'AD et la ChM de l'état de la mise en œuvre. Et elle charge la CdG de faire des rapports réguliers à la ChM sur l'avancement des travaux." La manière dont les choses se sont déroulées aujourd'hui pose problème à Lars Frauchiger/ASMAC. D'une part, il ne dispose pas, en tant que délégué, de l'intégralité des informations et des documents. D'autre part, on exige de lui qu'il se prononce, en l'espace de quelques minutes, sur un montant en millions qui engagera résolument la FMH dans une direction ou dans l'autre.

Thomas Heuberger/BE précise encore une fois que la plupart des interventions entendues, y compris l'avis exprimé par le conseiller juridique, vont dans la même direction. Cela exprime clairement que le projet a un sens, mais que les conséquences des différentes propositions ont été insuffisamment réfléchies et analysées. C'est aussi dans ce sens qu'il faut comprendre les deux propositions de renvoi de l'ASMAC et de la SMSR. Selon 
lui, la bonne solution consiste à charger le CC, en sa qualité d'organe exécutif, d'élaborer des propositions en tenant compte des conséquences qui en découlent.

Fiorenzo Caranzono/TI estime qu'il est irresponsable de se prononcer aujourd'hui sur des activités opérationnelles. La ChM est toutefois en droit de savoir sur quelle base le CC mènera ses réflexions et élaborera ses propositions. Il estime que la question à trancher porte sur la communication des conclusions de la Task force, et non sur la prise de décisions.

Jürg Schlup/président FMH clôt les débats relatifs à l'entrée en matière par cette intervention et charge la Task force de conclure.

\subsubsection{Intervention finale du Groupe de travail}

Peter Wiedersheim/Task force s'étonne de la façon dont les choses se déroulent aujourd'hui. La Task force a exécuté le mandat qui lui a été confié en son âme et conscience et la mandante est disposée à entendre ses conclusions. Il ne comprend pas que l'organe décisionnel bloque ainsi les informations et ne donne pas à la Task force la possibilité de présenter les résultats et les conclusions de son travail. Les interventions du CC le surprennent, ce d'autant plus qu'il est toujours parti du principe que leur collaboration était bonne. A titre d'exemple, il cite l'ancien projet «Modèles de direction», qui avait été accepté à $90 \%$ des voix. Malheureusement, ce projet est resté à ce jour au stade de l'adoption et n'a pas été mis en œuvre.

Idéalement, il souhaiterait que chaque responsable de département soit aux commandes des aspects stratégiques et sache qu'il a derrière lui une équipe parfaitement opérationnelle. Il considère que le CC a une fonction essentiellement stratégique. Il aspire à un travail d'équipe et croit dès lors à la ChM. Lorsqu'elle est unie, la FMH est forte, et il espère que tous contribueront de manière constructive à une FMH qui sera à l'avenir encore plus performante qu'aujourd'hui. C'est aussi l'objectif de la Task force. Il souhaite savoir si les délégués veulent entendre les conclusions du groupe d'experts. La responsabilité de la gestion de la mise en œuvre peut encore être discutée.

\subsection{Votes}

Le déroulement du vote est fixé comme suit:

- Proposition ASMAC versus proposition SMSR

- Proposition adoptée versus entrée en matière

La proposition de l'ASMAC est formulée comme suit:

1. Non-entrée en matière sur les propositions de stabilisation budgétaire de la Task force

2. Dissolution de la Task force

3. Mandat de la ChM au CC et au SG de la FMH les chargeant, après analyse du rapport de KPMG, de présenter à la prochaine ChM des propositions motivées ayant pour objectif de stabiliser le budget et de générer des gains d'efficience.

La proposition de la SMSR est formulée comme suit: La ChM prend connaissance du rapport de la Task force et le renvoie au CC afin qu'il l'analyse de manière approfondie en collaboration avec le SG. Le CC proposera à la ChM de l'automne 2015 des mesures réalistes qui seront intégrées au budget 2016.

\section{Décision:}

La proposition de la SMSR l'emporte avec 89 voix, contre 45 pour celle de l'ASMAC et 5 abstentions.

Josef Widler/AGZ demande que, pour le prochain vote relatif à l'entrée en matière, on vote par «oui» ou «non». Thomas Heuberger/BE fait remarquer que la formulation de la proposition de la SMSR est: "prend connaissance du rapport de la Task force». Or, on ne peut pas prendre connaissance de quelque chose qu'on n'a pas vu. Il ne souhaite toutefois pas de nouvelle discussion détaillée. Il propose donc à nouveau de voter.

La formulation de la proposition de la SMSR est ambiguë. Jürg Schlup/président FMH demande des précisions. Pierre-Alain Schneider/VD explique que la proposition stipule clairement qu'il convient de prendre connaissance du rapport. Le rapport qui a été demandé doit être fourni au CC. La ChM ne peut pas se prononcer sans disposer des informations nécessaires. Selon lui, «prendre connaissance» signifie qu'il faut procéder à la présentation.

Pierre Vallon/Task force demande à la SMSR s'il est question du rapport de KPMG dans son intégralité, ou uniquement de la présentation.

Pierre-Alain Schneider/VD répond qu'il s'agit de la présentation qui a été faite à l'AD.

Jürg Schlup/président FMH précise que sur la base de la proposition Gafner qui a déjà été votée, les délégués renoncent à une présentation en cas d'adoption de la proposition de l'ASMAC ou de celle de la SMSR. Compte tenu du fait que la proposition de la SMSR a été adoptée entre-temps, cela implique le renvoi au CC et la présentation de nouvelles propositions et mesures à la ChM du 29 octobre 2015. Par ce vote, il n'a pas été consenti à la présentation.

Hans-Anton Vogel/AR propose le réexamen de la question de la présentation.

Après une brève interruption, Jürg Schlup/président $F M H$ recommande une marche à suivre présentée par le Service juridique.

Hanspeter Kuhn/FMH propose trois votes:

1. Proposition de réexamen de Hans-Anton Vogel (nous voulons voir la présentation)

2. En cas d'adoption de la proposition de réexamen, il est possible de diviser la proposition de la SMSR de 


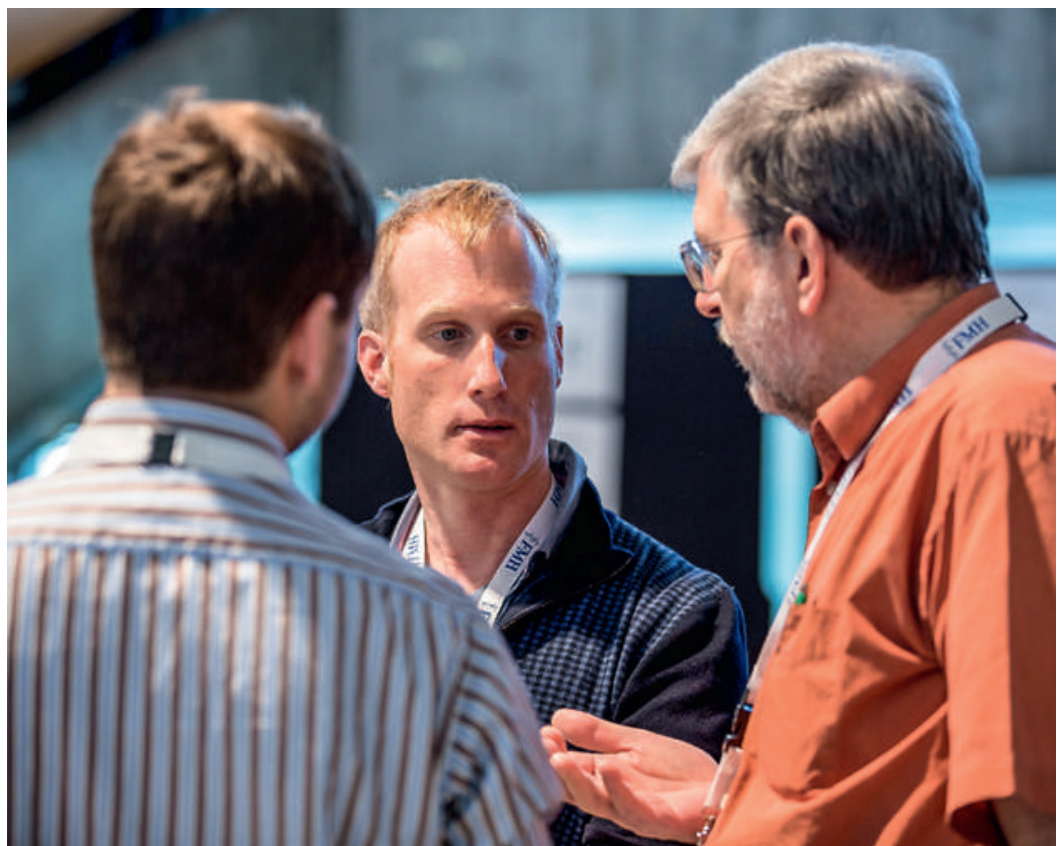

La Chambre médicale favorise le dialogue entre les générations.

manière à voter séparément sur les différents points. C'est ensuite le renvoi au CC qui sera soumis au vote.

3. Faut-il procéder à la présentation aujourd'hui même en cas de non-entrée en matière?

Konrad Schiess/SG estime que cette marche à suivre altère la procédure. S'il en est ainsi, il propose de ne voter qu'ultérieurement sur les propositions complémentaires et les présentations. Il propose de transférer le mandat à une commission - similaire à une commission parlementaire, qui présentera ensuite ses suggestions à la ChM.

Pour Hanspeter Kuhn/FMH, cette proposition est formulée tardivement. Il s'agit maintenant de voter sur l'entrée en matière ou la non-entrée en matière sur l'examen de détail. La SMSR propose le renvoi au CC, et Konrad Schiess propose le renvoi à une commission au sein de laquelle le CC serait également représenté. Si on entre en matière sur cette nouvelle proposition, elle devrait alors être opposée à celle de la SMSR. La proposition adoptée serait alors retenue.

Michaël Hagmann/VD propose de retenir la présentation dans tous les cas, que l'entrée en matière soit acceptée ou non.

Jürg Schlup/président FMH approuve cette manière de procéder. Les propositions sont soumises au vote:

Proposition de réexamen Vogel:

La proposition est formulée comme suit: nous voulons voir la présentation.

Décision:

La proposition de réexamen est acceptée par 117 voix contre 21 et 1 abstention.

\section{Proposition:}

Nous voulons voir la présentation dans tous les cas, que l'entrée en matière soit acceptée ou non.

\section{Décision:}

La proposition est acceptée par 110 voix contre 20 et 1 abstention.

Proposition SMSR:

Il n'est pas procédé à l'examen de détail, le dossier est renvoyé au CC conformément à la proposition de la SMSR, et il n'est pas entré en matière

\section{Décision:}

Cette proposition est acceptée par 107 voix contre 25 et 4 abstentions.

$\mathrm{Au}$ vu de ces votes, l'examen de détail est superflu. KPMG présentera néanmoins son rapport. L'acceptation de la proposition de la SMSR clarifie également la question du mandat de la Task force. Le dossier est transféré au CC.

Stefan Greuter/ASMAC dépose une motion d'ordre demandant de traiter les points à l'ordre du jour avant la présentation de KPMG.

\section{Motion d'ordre Greuter/ASMAC}

Les points 10 et 11 de l'ordre du jour doivent être traités avant la présentation.

\section{Décision:}

La motion d'ordre Greuter est acceptée par 103 voix contre 5 et 12 abstentions.

Pierre Vallon/Taskforce souhaite mettre fin aujourd'hui à son mandat au sein de la Task force. Au vu des circonstances, il n'entend pas poursuivre sa tâche.

\section{Révision globale du TARMED}

La révision globale du TARMED était un grand projet mené par Ernst; il s'en est chargé avec un grand soin et beaucoup d'engagement au cours des dernières années. Le projet est dans la dernière ligne droite et les luttes de répartition commencent. Urs Stoffel/CC FMH, qui a repris ce dossier d'Ernst, remercie à cette occasion l'équipe des "tarifs ambulatoires" à Olten, qui a infatigablement travaillé à cette révision avec un engagement hors du commun dans l'intérêt du corps médical.

Le projet «Révision globale du TARMED» a si bien avancé qu'il est possible de démarrer une consultation interne sur divers chapitres du point de vue de la technique tarifaire. Le processus de consultation présenté aujourd'hui concerne le déroulement de la consultation au niveau des équipes spécialisées et le processus interne à la FMH prévu pour traiter les résultats du travail de ces équipes. Tous les intéressés doivent avoir la possibilité de voir ouvertement et en toute transparence ce qui a changé et comment, et de prendre position à ce sujet. Processus 1: Niveau équipe spécialisée 
Léquipe spécialisée est chargée de mettre à jour la nomenclature (catalogue des prestations médicales) et, si nécessaire, l'infrastructure. Ce travail débouche sur le catalogue des prestations (minutage inclus) et divers compléments à l'infrastructure. Si les sociétés de discipline impliquées ne sont pas d'accord avec les propositions des partenaires de la révision, il y a deux possibilités. L'équipe spécialisée, dirigée par un représentant $\mathrm{H}+$, FMH ou CTM (partenaires de la révision), a pour mandat de transmettre une proposition commune à la commission technique (TeKo) des partenaires de la révision. En plus des représentants des sociétés de discipline concernées, c'est-à-dire celles qui facturent plus souvent que les autres dans un chapitre donné, des experts tarifaires des partenaires de la révision $\mathrm{H}+, \mathrm{FMH}$ et CTM sont également présents dans l'équipe spécialisée. Que se passe-t-il en cas de désaccord entre les partenaires de la révision, c'est-à-dire si les représentants de la FMH (sociétés de discipline et experts tarifaires) sont d'accord, mais soutiennent une autre proposition tarifaire que H+ ou la CTM, par exemple? Dans ce cas, les étapes de coordination sont clairement réglées dans le cadre du projet de révision. Tout d'abord au sein de la direction opérationnelle de la TeKo et ensuite dans la commission de pilotage. Tous les partenaires de la révision sont à nouveau présents dans ces deux organes et les voies de décision sont définies.

Que se passe-t-il en cas de désaccord au sein de la FMH? Pour pouvoir déposer une proposition commune, les représentants des partenaires de la révision (FMH, CTM, $\mathrm{H}+$ ) doivent être d'accord. S'il y a deux propositions ou plus au sein de la FMH, que ce soit par deux sociétés de discipline ou lorsque les représentants des sociétés de discipline et les experts tarifaires de la FMH ne parviennent pas à s'entendre sur une proposition commune, il s'agit de définir le processus permettant à la FMH de décider quelle proposition elle soutiendra dans l'équipe spécialisée. Le CC de la FMH est alors l'organe de coordination et de décision.

Si on ne parvient pas à s'entendre dans l'équipe spécialisée, il y a la possibilité interne à la FMH de soumettre les propositions au CC de la FMH ou celle de s'adresser aux partenaires de la révision. S'il y a ici des divergences, la TeKo et la commission de pilotage sont responsables pour parvenir à un accord. Et finalement, la proposition de révision est validée par l'équipe spécialisée.

Processus 2: Consultation externe (partenaires de la révision)

Après validation par l'équipe spécialisée, la proposition est vérifiée et approuvée par la TeKo.

Processus 3: Consultation interne à la FMH

Les sociétés de discipline sont informées des résultats et le navigateur est mis en ligne. Toutes les sociétés de discipline ont la possibilité de voir le chapitre révisé et de vérifier si la structure tarifaire présentée correspond aux principes de tarification et si elle reproduit leur quotidien médical.

Les délégués tarifaires ou la présidence d'une société de discipline collectent ensuite les réactions sur un chapitre publié et les transmettent à la FMH; celle-ci les fait à son tour parvenir à la société de discipline qui a élaboré la proposition pour qu'elle en prenne connaissance. Toute réclamation doit comprendre la raison de celleci, l'annonce des prestations manquantes ainsi que les solutions de rechange proposées, avec une motivation. S'il n'y a aucun besoin d'intervention, le processus 3 est terminé. En cas de désaccord, le CC prend une décision et transmet les données à la TeKo pour vérification. S'il n'y a aucune solution à ce niveau, la commission de pilotage prend une décision et la phase d'évaluation est terminée.

Les différentes remarques dans le cadre du processus de consultation interne à la FMH ne reçoivent pas de réponses individuelles; les réponses, motifs et décisions sont publiés et communiqués à l'intention de toutes les organisations concernées.

Processus 4: Consolidation / travaux finaux

Après la fin du processus 3, le navigateur sera mis à jour. D'éventuelles adaptations (relevant de la technique tarifaire) pourront toujours être introduites. La révision technique de la structure sera ainsi terminée et la structure technique pourra alors être dévoilée au public.

Ce processus a été présenté et discuté tant au CC qu'à l'AD et également lors de la Journée des délégués tarifaires.

La prochaine étape sera d'informer les délégués tarifaires et les présidents du démarrage du processus 3 de la consultation. L'objectif est de pouvoir soumettre les conventions-cadres ainsi que la structure tarifaire complète à l'approbation de la ChM du 29 octobre 2015. Michel Matter/GE veut savoir si la question de la neutralité des coûts sera à nouveau d'actualité en cas de changement de modèle. Selon la convention en vigueur, c'est ce qui est prévu.

Urs Stoffel/CC FMH signale que cette question était l'un des thèmes principaux de la Journée des délégués tarifaires du 6 mai 2015. Ce qui a été présenté hier a montré que la révision d'une structure tarifaire, tant qu'on ne change pas le modèle sur lequel elle repose, est en fait une révision et non un changement de modèle tarifaire. Or, pour la révision de la structure tarifaire, rien n'a été changé au modèle. C'est pourquoi, il est clair pour la FMH que l'art. 59c, al. 1c, de l'Ordonnance sur l'assurancemaladie (seule base légale exigeant cette neutralité des coûts) ne s'applique pas. La FMH maintiendra donc cette position. 
Philippe Rheiner/GE a une question sur le processus 2. Que se passe-t-il si la commission technique n'accepte pas la proposition? Est-elle renvoyée à la société de discipline, qui prend alors la décision?

Urs Stoffel/CC FMH répond que la société de discipline médicale est consultée encore une fois avant la décision définitive.

Les délégués votent séparément sur deux propositions. Proposition:

Approbation du processus de consultation technique de la structure tarifaire conformément au chiffre 2 de la circulaire

Décision:

La proposition est acceptée par 153 oui, avec 4 abstentions.

Proposition:

Le CC de la FMH est l'organe de coordination et de décision pour la consultation interne à la FMH.

Décision:

La proposition est acceptée par 145 oui contre 1 non, avec 7 abstentions.

\section{Rapport sur la HPC}

La collaboration de la FMH avec Swisscom, fournisseur de la HPC, prend fin le 30 juin 2015. A partir de 2016, Swisscom ne proposera plus de services de certificats embarqués sur une carte. Urs Stoffel/CC FMH informe que le CC a saisi cette opportunité pour reconsidérer sa stratégie, notamment pour les identités électroniques (eID). Selon diverses enquêtes (baromètre eHealth) et d'après les expériences réalisées avec les projets-pilotes d'eHealth («Mon dossier médical»), l'avenir ne réside pas dans les eID sur des cartes en tant que médias. La FMH opte désormais pour une stratégie «multichannel». Le projet HPC 2.0 comprend quatre niveaux de restructuration et le $\mathrm{CC}$ a déjà autorisé la réalisation des niveaux 1 et 2 . Ces niveaux de restructuration prévoient la fin de la HPC fournie par Swisscom au 30 juin 2015 et sa reprise par HIN, propre fournisseur de la FMH, en collaboration avec QuoVadis au $1^{\text {er }}$ juillet 2015. Il n'y a aucun risque pour l'exploitation des cartes HPC existantes. Dans une deuxième phase (niveau 3 et 4), qui aura lieu en 2016, il s'agira pour HIN de mettre sur pied un système d'eID ne se fondant pas sur une carte mais sur une solution Client sur PC, avec enregistrement sur un serveur hautement sécurisé et accès via le «cloud».

QuoVadis, l'une des quatre entreprises pouvant émettre des certificats, a été choisie comme nouveau fournisseur. Le passage du certificat embarqué sur une carte à la nouvelle stratégie "multichannel» permettra de réduire sensiblement les coûts d'exploitation de la HPC à moyen et long terme. Vu les exigences techniques complexes pour le changement de fournisseur, un retard d'un à deux mois est possible pour les nouvelles commandes de cartes HPC avec certificat. Les certificats qui sont en circulation et fonctionnent ne risquent rien. Mais, pour ce qui concerne les nouveaux certificats, il se peut qu'ils ne fonctionnent que dès la fin août de cette année.

Personne ne demande la parole et les délégués prennent connaissance de ces informations.

\section{IPI}

\subsection{Activités 2015}

Anne-Geneviève Bütikofer/SG FMH informe la ChM de la situation contractuelle et de l'état des activités en 2015. Après l'acceptation de la contribution spéciale lors de la ChM d'octobre 2013, le contrat de prestations a été signé en février 2014. Le 30 octobre 2014, la ChM a décidé de reconduire le contrat de prestations mais pour une année seulement. Aucun avenant 2015 au contrat de prestations 2014 n'a encore été conclu à ce jour entre l'IPI et la FMH, car les annexes (qui font partie intégrante de l'avenant) sont lacunaires. Le tableau de bord notamment fait encore défaut malgré plusieurs interventions. En revanche, seuls 5 projets sont planifiés par l'IPI en 2015, contre 17 en 2014. Le business plan et le plan financier pour les années 2015 à 2017 ont entretemps été remis.

Le groupe de travail eHealth est informé de la situation actuelle et soutient la démarche du CC consistant à exiger les documents qui font encore défaut. En conclusion, la FMH n'est pas aujourd'hui en relation contractuelle avec l'IPI. La cotisation extraordinaire a cependant été prélevée. Un paiement à l'IPI, en revanche, ne pourra avoir lieu qu'après la remise des pièces complètes au Secrétariat général de la FMH. Aujourd'hui, il n'est pas possible de rendre compte d'autres activités.

Hans-Anton Vogel/VEDAG demande qu'on ne perde pas le sens de la mesure concernant les exigences administratives. L'IPI dispose de trop peu de personnel pour qu'une grande partie de ses ressources soit utilisée à cet effet. Le directeur fait un très bon travail et il conviendrait de le reconnaître. L'IPI a une tâche extrêmement importante pour l'avenir et il ne faudrait pas le condamner à l'échec par un excès de charges administratives.

Anne-Geneviève Bütikofer/SG de la FMH prend ces remarques au sérieux et souligne que les documents manquants génèrent aussi une surcharge de travail pour le Secrétariat général. Ce dernier a pour tâche et obligation de vérifier l'exécution du contrat de prestations et l'utilisation des moyens alloués. Or, d'un point 
de vue opérationnel, il n'est pas possible de le faire pour l'instant, vu l'absence de documents importants. Si les organes décident de poursuivre les activités de l'IPI sous l'angle purement politique, c'est une décision politique mais pas une décision opérationnelle.

Jean-Pierre Pavillon/VD souhaiterait savoir si l'IPI a fourni à ce jour un travail concret utilisable.

Anne-Geneviève Bütikofer/SG de la FMH confirme la collaboration constructive, mais précise que l'exécution des tâches administratives fait aussi partie d'une bonne gestion d'entreprise. Daniel Schröpfer/ASMAC souligne que l'ASMAC soutient les projets de l'IPI et rappelle que la ChM vient de voter qu'un contrat de prestations doit être conclu pour toute prestation de plus de CHF 10000 . Il se demande qui, dans le libre marché, reçoit près de CHF 1 million par an sans devoir rendre des comptes.

Anne-Geneviève Bütikofer/SG de la FMH pense que pour un montant aussi conséquent, il est indispensable d'effectuer un suivi des travaux. Le «balance score card» est un outil essentiel pour examiner les objectifs et la réalisation des étapes. Le Secrétariat général a besoin de ce document pour pouvoir comprendre où l'argent alloué est utilisé.

Hans-Anton Vogel/VEDAG rappelle la décision de la ChM d'intégrer l'IPI dans le département eHealth. Pour lui, peu d'avancées concrètes sont visibles à ce propos. Ce devrait pourtant être la première tâche, au lieu de la conclusion de ce contrat. Il demande encore une fois de soutenir l'IPI.

Anne-Geneviève Bütikofer/SG de la FMH comprend cette préoccupation, mais fait remarquer qu'il s'agit là de deux choses différentes: d'une part il y a les activités de l'IPI pour l'année 2015 et, d'autre part, son intégration dans le département eHealth prévue pour 2016. Pour le Secrétariat général, il est logique de faire un rapport à la ChM.

Gerhard Schilling/président IPI prend position sur les documents manquants. Il informe les délégués du travail effectif du directeur, qui doit consacrer $40 \%$ de son temps de travail rien que pour l'élaboration des documents administratifs. Il rend compte de l'état des projets actuels et de la dispensation des cours "going paperless». L'IPI participe actuellement à de nombreux projets, ce qui constitue en soi un succès. Il prie les délégués de reconnaître ce travail et de ne pas surcharger l'IPI avec des questions mineures.

Pour Daniel Schröpfer/ASMAC, la question du tableau de bord n'est pas une question mineure. Encore une fois: qui, aujourd'hui, reçoit un crédit de CHF 1 million sans devoir se justifier?

Andreas Häfeli/ZH avait déjà un mauvais pressentiment lors du vote d'aujourd'hui sur les contrats de prestations, surtout concernant la procédure. Comme il n'a pas pris la parole à ce moment-là, il prie le CC de vérifier le diagramme de flux et la façon de procéder. On ne peut pas se permettre de bloquer la conclusion d'un contrat au niveau du Secrétariat général pour des raisons formelles. Anne-Geneviève Bütikofer/SG de la FMH conclut qu'il n'est en aucun cas question de mettre des barrières sur la route de l'IPI. Mais elle rappelle que le SG porte la responsabilité des données budgétaires. Et, compte tenu de la situation financière actuelle de la FMH, il est nécessaire de faire preuve d'un peu de formalisme et de discipline.

\subsection{Intégration de l'IPI dans le département eHealth de la FMH}

Revenant sur la discussion précédente, Urs Stoffel/CC $F M H$ soutient aussi bien l'IPI dans ses efforts que le SG FMH, qui réclame les documents manquants pour pouvoir conclure un nouveau contrat de prestations. S'agissant de l'intégration de l'IPI dans le département eHealth, le groupe de travail eHealth, dans lequel l'IPI est également représenté, a entre-temps discuté différents modèles et examiné des modèles existants. Le département eHealth propose un modèle analogue à celui de l'ASQM, qui pourrait être repris tel quel. L'organisation doit avoir des structures simples, utiliser les synergies et éviter le travail à double. L'«Advisory Board» (comité consultatif) IPI est un «réservoir» qui contient notamment l'expérience des fondateurs de l'IPI. L'IPI ne continuera pas sous sa forme actuelle. Le nouveau nom devra faire penser à un nouveau départ. L'organe de direction, tel qu'il est présenté ici, a été salué par le groupe de travail eHealth. Le développement du modèle sera maintenant poursuivi, raison pour laquelle le groupe de travail souhaiterait obtenir aujourd'hui l'approbation de la ChM, afin d'accélérer l'intégration de l'IPI dans le département eHealth.

Daniel Schröpfer/ASMAC veut savoir pourquoi on a encore besoin d'un centre pour l'informatique au cabinet médical, s'il est sous la direction d'eHealth FMH. Ce terme prête à confusion.

Urs Stoffel/CC FMH explique que cette proposition est un travail en cours et que l'organigramme final avec les termes définitifs sera présenté à la ChM en automne.

Jürg Nadig/SSOM fait remarquer qu'il y a une différence entre les modèles présentés. A son avis, dans l'ASQM, toutes les sociétés de discipline sont représentées, tandis que dans le groupe de travail eHealth, seulement les associations faîtières le sont. Pour lui, la question se pose de savoir si les sociétés de discipline peuvent s'investir suffisamment dans ce modèle.

Urs Stoffel/CC FMH signale que le groupe de travail eHealth ne comprend pas que les associations faîtières, 
mais aussi les sociétés de discipline. Si seule l'association faîtière devait être représentée, la base pourrait bien entendu également siéger.

Quant à la question du financement, Urs Stoffel/CC FMH explique qu'une cotisation extraordinaire est validée jusqu'à la fin 2015. En ce qui concerne l'intégration dans le département eHealth, il s'agit d'un nouveau mandat et d'une nouvelle tâche. Le département eHealth propose soit de budgétiser les nouvelles tâches et d'augmenter le budget ordinaire, soit d'augmenter la cotisation de membre ordinaire, au lieu de demander une cotisation extraordinaire.

Adrian Sury/président CdG rappelle que la CdG a déjà insisté plusieurs fois sur le fait qu'il ne faut pas financer les dépenses récurrentes par une contribution spéciale. Les contributions spéciales ne servent qu'à des fins extraordinaires. Il souhaite qu'on supprime la contribution spéciale liée de la proposition.

Hanspeter Kuhn/FMH rappelle la disposition sur les contributions spéciales qui figure à l'art. 11, al. 3, des Statuts et dont les termes sont les suivants:

${ }^{3}$ En tenant compte du principe du bénéficiaire-payeur, la FMH et les SCM fixent les cotisations annuelles suivantes: - la cotisation de base générale annuelle, les éventuelles contributions spéciales pour certains groupes de membres ou des projets déterminés. Le montant de la cotisation de base est fixé en fonction du statut professionnel du membre. Le Règlement d'exécution définit les différentes catégories, de même que les critères de réduction des cotisations.

Daniel Schröpfer/ASMAC ne voit pas pourquoi on devrait maintenant, au point 2 , prendre une décision sur le financement. En fait, cette décision ne devrait être prévue qu'en automne 2015.

Pour Urs Stoffel/CC FMH, une décision signifie que les délégués sont d'accord d'examiner les deux possibilités de financement. Si les délégués se prononcent en faveur d'une variante, seule celle-ci sera poursuivie.

Hans-Jakob Riedtmann/ZH rappelle qu'avant ce point de l'ordre du jour, lors de la stabilisation du budget, on a parlé de fixer une marge de manœuvre opérationnelle. Le CC présentera à la ChM d'octobre 2015 quelles ressources humaines et financières seront libérées pour cette marge de manœuvre opérationnelle. Si les délégués tiennent vraiment à l'IPI, il sera alors possible de fixer des priorités pour les ressources libérées. Il n'est donc pas nécessaire d'augmenter la cotisation de membre ordinaire.

Les deux propositions sont soumises au vote comme suit:

Proposition du CC:

Poursuite de l'intégration de l'IPI dans le département eHealth et dans les structures de la FMH selon le modèle proposé de l'ASQM, conformément au mandat de la Chambre médicale du 30 octobre 2014.

Décision:

La proposition est acceptée par 104 oui contre 9 non, avec 11 abstentions.

Proposition du CC:

Décision d'une contribution spéciale liée ou d'une augmentation de la cotisation de base pour financer le mandat supplémentaire concernant l'informatique au cabinet.

\section{Décision:}

La proposition est rejetée avec 32 oui, 57 non et 25 abstentions.

Selon Urs Stoffel/CC FMH, cette décision implique de prévoir le financement dans le budget ordinaire, sans augmentation du budget, et sans contribution spéciale ni augmentation de la cotisation de membre.

\section{Présentation du rapport de la Task force par la société KPMG}

Comme il en a été décidé au point 8 de l'ordre du jour, le Dr Walter Gratzer/KPMG a la possibilité de présenter le rapport des résultats de la Task force.

Conformément au mandat et à la décision de la $\mathrm{ChM}$, la Task force a demandé à la société KPMG de procéder à une analyse de base. Dans un premier temps, chaque prestation fournie par la FMH a été analysée et évaluée. Dans un deuxième temps, 24 entretiens ont été menés avec des collaborateurs de la FMH, autrement dit avec l'ensemble des membres du Comité central, du SG et tous les cadres. Des analyses comparatives avec des tiers externes, p. ex. dans le secteur informatique, ont également été effectuées là où cela semblait pertinent et utile aux objectifs visés. Cette démarche a permis d'élaborer des trains de mesures et de mettre en lumière les potentialités. Le rapport a ensuite été présenté à l'AD, au CC et aux collaboratrices et collaborateurs de la FMH. Pour la Task force, il était essentiel de procéder à une analyse très fouillée et différenciée, tant au niveau des divisions que des sujets traités. Celle-ci a permis d'identifier des potentialités, notamment concernant l'exigence de dégager une marge de manœuvre opérationnelle de CHF 4-6 millions. Des «quick wins» ou mesures à effets bénéfiques immédiats ont également été identifiés. Aucune suppression de poste n'est prévue. La proposition vise plutôt une réaffectation des effectifs afin de libérer des ressources pour de nouveaux projets et de nouvelles initiatives permettant de renforcer la FMH dans son ensemble.

Deux approches différentes sont possibles pour améliorer les résultats, soit réduire les charges, soit augmenter les produits. Pour la première, il est possible d'aug- 
menter les recettes, d'améliorer les marges ou de créer des recettes additionnelles, mais aucune potentialité substantielle n'a pu être identifiée dans ce domaine, l'accroissement des recettes se basant exclusivement sur l'augmentation des cotisations de membres. Pour la deuxième, un potentiel d'économies a été identifié au niveau des frais matériels et des frais de personnel.

Le Dr Walter Gratzer/KPMG présente les potentialités identifiées qui reposent principalement sur la modification du portefeuille des prestations, l'optimisation du portefeuille de projets, l'optimisation des processus et l'optimisation en matière d'internalisation / d'externalisation. La mise en œuvre des mesures n'implique, en principe, pas de suppression de postes, étant donné que la durée de réalisation est prévue sur 3 ans et demi. Un redimensionnement du CC, la création de 6 centres de compétence et la prise de mesures transversales telles que la prise en charge des indemnités de séance des membres de la ChM par les sociétés délégantes, l'optimisation de la HPC par le changement de partenaire, la réduction du nombre annuel d'assemblées des délégués de 6 à 5 et la fermeture du site d'Olten, sont d'autres propositions à évaluer.

Etant donné que le CC est désormais compétent pour la poursuite des travaux, il n'est pas nécessaire de présenter le calendrier de la mise en œuvre et d'expliquer les propositions de décision. Il incombe désormais au CC et au SG de définir le calendrier d'application des mesures. En tant qu'entreprise externe à la FMH, KPMG a procédé à une analyse de la situation d'un point de vue externe tout en intégrant la vision interne. Le Dr Walter Gratzer/ KPMG, fait remarquer qu'au sein de la FMH comme dans toute autre organisation d'ailleurs, des intérêts et avis différents ont cours, ce qui fait que diverses approches peuvent être choisies pour réaliser l'une ou l'autre mesure. Il recommande de mettre en œuvre un processus clair et structuré, de sorte à ce que les choses puissent se développer de manière équilibrée et mener à des propositions sérieuses. Il remercie les délégués d'avoir eu la possibilité de présenter son rapport et la FMH pour la bonne collaboration.
Jürg Schlup/président FMH remercie le Dr Walter Gratzer pour ses explications. Il est persuadé que sur la base de ce rapport, le CC proposera cet automne à la ChM des mesures applicables. Il saisit l'occasion pour remercier également les membres de la Task force, en particulier Peter Wiedersheim et Adrian Sury, pour leur précieuse collaboration qui a impliqué une importante charge de travail supplémentaire.

\section{Informations du CC, de l'ISFM, du SG et des départements}

\subsection{Développement de la marque: remaniement du logo}

Les délégués ont reçu des documents de séance à ce propos. Personne ne pose de question ni ne demande la parole.

\subsection{Questions aux membres du Comité central} Pas d'autre question aux membres du CC.

\section{Divers}

\subsection{Plan des séances de la FMH pour 2016}

Les délégués prennent acte des dates de séance 2016 décidées au CC et à l'AD. Si la date de cette manifestation d'envergure devait coïncider avec d'autres engagements fixes pour 2016, la secrétaire générale invite les délégués à en informer le secrétariat central. En 2016, les séances de la Chambre médicale auront lieu le 26 mai et le 27 octobre.

La Chambre médicale se termine à l'heure prévue. Jurg Schlup remercie tous les délégués pour leur engagement et leur collaboration constructive et leur souhaite un bon retour. Il les informe également que vu les points traités, la séance extraordinaire prévue le 3 juin 2015 n’aura pas lieu. La prochaine séance de la Chambre médicale sera donc celle du 29 octobre 2015. 


\section{Glossaire}

\begin{tabular}{|c|c|}
\hline AD & Assemblée des délégués \\
\hline AeG & Société des médecins du canton de Soleure \\
\hline AGZ & Société des médecins du canton de Zurich \\
\hline AMDHS & Association des médecins dirigeants d'hôpitaux de Suisse \\
\hline ASMAC & Association suisse des médecins-assistants et chefs de clinique \\
\hline ASQM & Académie suisse pour la qualité en médecine \\
\hline ASSM & Académie suisse des sciences médicales \\
\hline BEKAG & Société des médecins du canton de Berne \\
\hline BMS & Bulletin des médecins suisses \\
\hline $\mathrm{CC}$ & Comité central \\
\hline CCDJP & Conférence des directrices et directeurs des départements cantonaux de justice et de police \\
\hline $\mathrm{CCs}$ & Code civil suisse \\
\hline CdG & Commission de gestion \\
\hline CDS & Conférence suisse des directrices et directeurs cantonaux de la santé \\
\hline ChM & Chambre médicale \\
\hline CNPT & Commission nationale de prévention de la torture \\
\hline CPC & Code de procédure civile \\
\hline CTM & Commission des tarifs médicaux LAA \\
\hline eHealth & Cybersanté, terme générique désignant l'ensemble des services de santé électroniques \\
\hline elD & Identité électronique \\
\hline EMH & Editions médicales suisses SA \\
\hline $\mathrm{FMH}$ & Fédération des médecins suisses \\
\hline FMPP & Foederatio Medicorum Psychiatricorum et Psychotherapeuticorum \\
\hline $\mathrm{H}+$ & Association des hôpitaux suisses \\
\hline HIN & Health Info Net SA \\
\hline HPC & Health Professional Card \\
\hline IPI & Institut pour l'informatique au cabinet \\
\hline ISFM & Institut suisse pour la formation médicale postgraduée et continue \\
\hline LPMéd & Loi sur les professions médicales \\
\hline MFE & Association des médecins de famille et de l'enfance Suisse \\
\hline PA & Loi fédérale sur la procédure administrative \\
\hline RE & Règlement d'exécution \\
\hline SFSM & Swiss Federation of Specialistes in Medicine \\
\hline SG & Secrétaire générale, Secrétariat général \\
\hline SMCB & Société des médecins du canton de Berne \\
\hline SMSR & Société médicale de la Suisse romande \\
\hline SSMI & Société suisse de médecine interne générale \\
\hline SSOM & Société suisse d'oncologie médicale \\
\hline SSU & Société suisse d'urologie \\
\hline TARMED & Tarif médical pour les prestations ambulatoires \\
\hline TeKo & Commission technique \\
\hline VEDAG & Association des sociétés médicales de Suisse alémanique \\
\hline
\end{tabular}

